\title{
Nonasymptotic Densities for Shape Reconstruction
}

\author{
Sharif Ibrahim, ${ }^{1}$ Kevin Sonnanburg, ${ }^{2}$ Thomas J. Asaki, ${ }^{1}$ and Kevin R. Vixie ${ }^{1}$ \\ ${ }^{1}$ Department of Mathematics, Washington State University, Pullman, WA 99164-3113, USA \\ ${ }^{2}$ Department of Mathematics, University of Tennessee Knoxville, Knoxville, TN 37996-1320, USA
}

Correspondence should be addressed to Sharif Ibrahim; math.densities@sharifibrahim.com

Received 17 August 2013; Revised 25 February 2014; Accepted 25 February 2014; Published 20 May 2014

Academic Editor: Victor Kovtunenko

Copyright (C) 2014 Sharif Ibrahim et al. This is an open access article distributed under the Creative Commons Attribution License, which permits unrestricted use, distribution, and reproduction in any medium, provided the original work is properly cited.

\begin{abstract}
In this work, we study the problem of reconstructing shapes from simple nonasymptotic densities measured only along shape boundaries. The particular density we study is also known as the integral area invariant and corresponds to the area of a disk centered on the boundary that is also inside the shape. It is easy to show uniqueness when these densities are known for all radii in a neighborhood of $r=0$, but much less straightforward when we assume that we only know the area invariant and its derivatives for only one $r>0$. We present variations of uniqueness results for reconstruction (modulo translation and rotation) of polygons and (a dense set of) smooth curves under certain regularity conditions.
\end{abstract}

\section{Introduction}

This work discusses the integral area invariant introduced by Manay et al. [1], particularly with regard to reconstructability of shapes. This topic has been considered previously by Fidler et al. $[2,3]$ for the case of star-shaped regions. Recent results have shown local injectivity in the neighborhood of a circle [4] and for graphs in a neighborhood of constant functions [5].

The present work does not assume a star-shaped condition but does make use of a tangent-cone graph-like condition which is local to the integral area circle. We also present an interpretation of the integral area invariant as a nonasymptotic density. This is based on a poster presented by the authors [6].

Our tangentially graph-like and tangent-cone graph-like conditions (Definitions 5 and 7 in Section 2) restrict our attention to shapes with boundaries that can locally (i.e., within radius $r$ ) be viewed as graphs of functions in a Cartesian plane in one particular orientation (in the case of tangentially graph-like) or a particular set of orientations (for tangent-cone graph-like). Intuitively, these conditions guarantee that the boundary does not turn too sharply within the given radius and that working locally in Euclidean space is the same as working locally on the boundary of our shapes (i.e., the shape boundary does not pass through any given invariant circle multiple times, Section 2.2). These simplifying assumptions allow us to explicitly analyze what happens when we move along the boundary and to work locally without worrying about global effects.

We show that the tangent-cone graph-like property can be preserved when approximating a shape with a polygon (Section 3) and discuss what the derivatives of these nonasymptotic densities represent (Section 4) and show that all tangentially graph-like boundaries can be reconstructed (modulo translations and rotations) given sufficient information about the nonasymptotic density and its derivatives (Section 5 and Appendix A).

The main contribution of this paper is to show (under our tangent-cone graph-like condition) that all polygons (Theorem 29 in Section 6) and a $C^{1}$-dense set of $C^{2}$ boundaries (Theorem 30 in Section 7) are reconstructible (modulo translations and rotations). We briefly discuss and sketch the proofs of these two theorems.

Theorem 1. For a polygon $\Omega$ which is tangent-cone graph-like with radius $r$, suppose that one has the integral area invariant $g(s, r)$ where $s$ is parameterized by arc length. Suppose that for all s one knows $g(s, r)$ and its first derivatives with respect to $r$ (disk radius) and $s$ (position along the boundary). This 
information is sufficient to completely determine $\Omega$ up to translation and rotation; that is, one can recover the side lengths and angles of $\Omega$.

The proof of this theorem uses the discontinuities in the $s$ derivative to determine the locations of vertices (and thus the side lengths between them). We combine the $r$ derivative and the one-sided $s$ derivative information when centered on a vertex to recover the angles at which the polygon enters and exits the circle (which might not be the polygon vertex angle if the circle contains another vertex). Doing this with the other one-sided $s$ derivative gives the same thing but using the orientation determined by the other polygon side incident to the vertex. The combination of these yields the polygon's angle at each vertex.

Theorem 2. Define $\mathbb{G} \equiv\left\{\gamma \mid \gamma\right.$ is a $C^{2}$ simple closed curve and tangentially graph-like for $r=\widehat{r}$ \}. Suppose that, for $r=\widehat{r}$, for all $s \in[0, L]$, and for each $\gamma \in \mathbb{G}$, one knows the first-, second-, and third-order partial derivatives of $g_{\gamma}(s, r)$. Then the set of reconstructible $\gamma \in \mathbb{G}$ is $C^{1}$ dense in $\mathbb{G}$ where reconstructability is modulo reparametrization, translation, and rotation.

The first part of the proof shows that the derivative information can be used to obtain the curvature. However, it is not the curvature at the boundary point where the circle is centered but rather the curvature at each of the points where the boundary enters and exits the circle. Although the Euclidean distance to these points is known, the arc length distances are not and can vary from point to point. Thus the sequences of curvatures we obtain also lose the arc length parameterization of our area invariant. The rest of the proof is concerned with finding the arc length distance from the center to the entry and exit points which effectively recovers the curvature for all points. This relies on matching up the unique features of exit angle sequences with each other which in turn relies on the existence of unique maxima and minima in these sequences. While this is not true in general, it can be arranged to be so by a suitable small perturbation of the boundary (which is why our result is one of density rather than for all shapes).

This is a theoretical paper about a measure that is useful in applications: we do not pretend that the reconstruction techniques in our proofs are practically useful. In fact, the reconstructions we use to show uniqueness would be seriously disturbed by the noise that any practical application would encounter. We do, however, comment on some possible approaches to reconstruction (Section 8) using the ORTHOMADS direct search algorithm [7] to successfully reconstruct shapes which are not predicted by our theory.

\section{Notation and Preliminaries}

Unless otherwise specified, we will be assuming throughout this paper that $\Omega \subset \mathbb{R}^{2}$ is a compact set with simple closed, piecewise continuously differentiable boundary $\partial \Omega$ of length $L$. Let $\gamma:[0, L] \rightarrow \partial \Omega$ be a continuous arclength parameterization of $\partial \Omega$ (see Figure 1). We will adopt

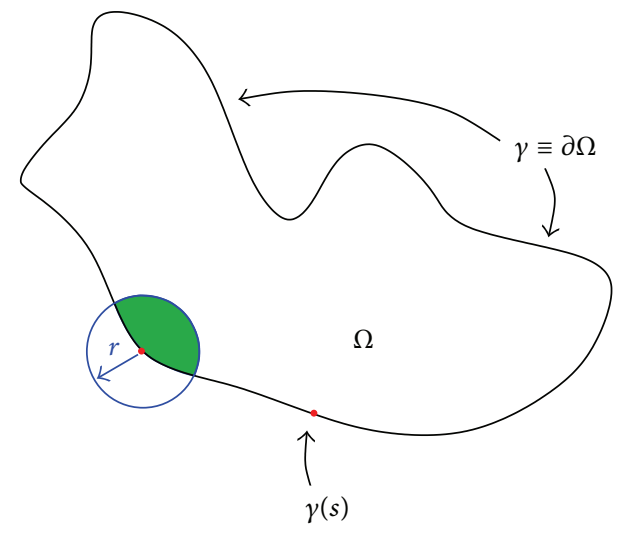

FIgURE 1: Notation and basic setup.

the convention that $\gamma$ traverses $\partial \Omega$ in a counterclockwise direction so it always keeps the interior of $\Omega$ on the left (there is no compelling reason for this particular choice, but adopting a consistent convention allows us to avoid some ambiguities later). Note that $\gamma(0)=\gamma(L)$ and that $\gamma$ restricted to $[0, L)$ is a bijection. Denote by $D(p, r)$ the closed disk and $C(p, r)$ the circle of radius $r$ centered at the point $p \in \mathbb{R}^{2}$.

In geometric measure theory, the $m$-dimensional density of a set $A \subseteq \mathbb{R}^{n}$ at a point $p \in \mathbb{R}^{n}$ is given by

$$
\Theta^{m}(A, p)=\lim _{r \downarrow 0} \frac{\mathscr{H}^{m}(A \cap D(p, r))}{\alpha_{m} r^{m}},
$$

where $\mathscr{H}^{m}$ is the $m$-dimensional Hausdorff measure and $\alpha_{m}$ is the volume of the unit ball in $\mathbb{R}^{m}$ [8]. In the current context, the 2-dimensional density of $\Omega$ at $\gamma(s)$ is simply

$$
\Theta^{2}(\Omega, \gamma(s))=\lim _{r \downarrow 0} \frac{\operatorname{Area}(\Omega \cap D(\gamma(s), r))}{\pi r^{2}} .
$$

While we can evaluate this for all $s \in[0, L)$, just knowing the density at every point along the boundary is generally insufficient to reconstruct the original shape. If $\gamma^{\prime}(s)$ exists, then $\operatorname{Area}(\Omega \cap D(\gamma(s), r))$ is approximated arbitrarily well for sufficiently small $r$ by replacing $\partial \Omega$ with its tangent line (which gives us an area of exactly $\pi r^{2} / 2$ ). Hence, we have $\Theta^{2}(\Omega, \gamma(s))=1 / 2$ at any point where $\gamma$ is differentiable. That is, just knowing $\Theta^{2}$ (i.e., the limit) is insufficient to distinguish any two shapes with $C^{1}$ boundary.

Contrast this with the situation where we know $\operatorname{Area}(\Omega \cap$ $D(\gamma(s), r))$ for every $s \in[0, L)$ and $r>0$ (i.e., we have all of the values needed to compute the limit as well). This added information is sufficient to uniquely identify $C^{2}$ curves by recovering their curvature at every point (see Appendix A).

One natural question to ask (and the focus of the present work) is whether failing to pass to the limit (i.e., using some fixed radius $r$ instead of the limit or all $r>0$ ) and collecting the values for all points along the boundary preserves enough information to reconstruct the original shape. That is, can a nonasymptotic density (perhaps along with information about its derivatives) be used as a signature for shapes? 


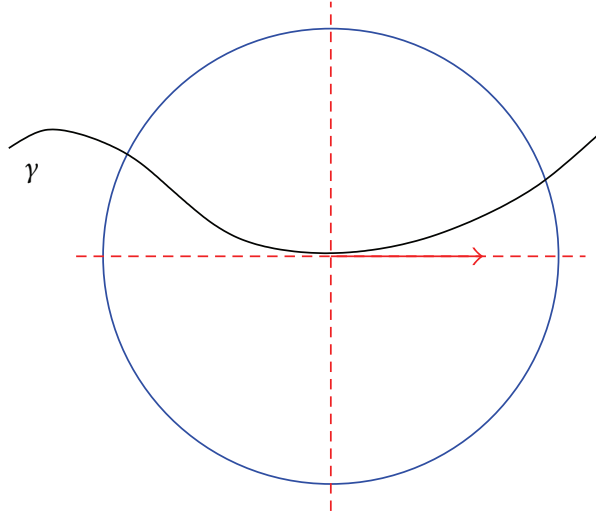

(a)

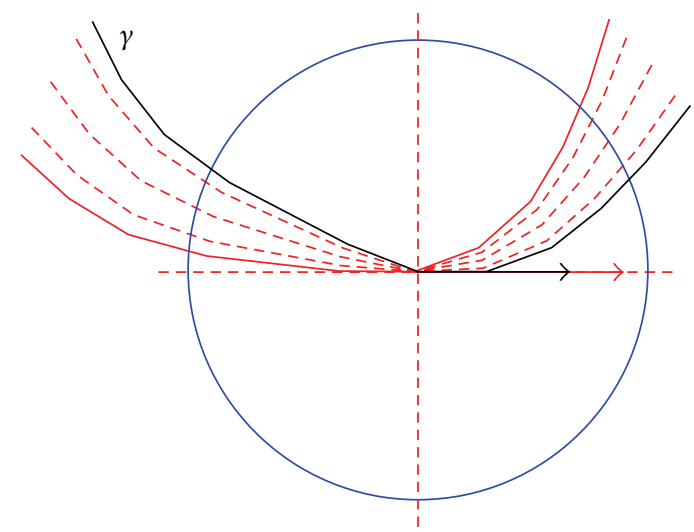

(b)

Figure 2: (a) Tangentially and (b) tangent-cone graph-like.

\subsection{Definitions}

Definition 3. In the current context, the integral area invariant $[1]$ is denoted by $g:[0, L) \times \mathbb{R}^{+} \rightarrow \mathbb{R}^{+}$and given by

$$
g(s, r)=\int_{D(\gamma(s), r) \cap \Omega} d x=\operatorname{Area}(\Omega \cap D(\gamma(s), r))
$$

Remark 4. Note the lack of the normalizing factor $\pi r^{2}$ in the definition of $g(s, r)$. Since we presume that $r$ is fixed and known for the situations we study, it is trivial to convert data between the forms $g(s, r)$ and $g(s, r) / \pi r^{2}$; we choose to leave out the normalizing factor in the definition of $g(s, r)$ as it is the integral area invariant of Manay et al. [1] and this form proves useful when computing derivatives in Section 4.

We introduce the tangentially graph-like condition as a simplifying assumption for the shapes we consider.

Definition 5. For a fixed radius $r$, one says that $\partial \Omega$ is graphlike (GL) at a point $p \in \partial \Omega$ (or graph-like on $D(p, r)$ ) if it is possible to impose a Cartesian coordinate system such that the set of points $\partial \Omega \cap D(p, r)$ is the graph of some function $f$ in this coordinate system. Without loss of generality, one adopts the convention that $p$ is the origin so that $f(0)=0$. One defines tangentially graph-like (TGL) in the same way but further requires that $\partial \Omega$ be continuously differentiable and $f^{\prime}(0)=0$ (noting that $f$ is $C^{1}$ because $\partial \Omega$ is). This is illustrated in Figure 2(a). Without loss of generality (and in keeping with our convention that $\gamma$ traverses $\partial \Omega$ counterclockwise), one assumes that the interior of $\Omega$ is "up" in the circle (i.e., $(0, \epsilon) \in \Omega$ for sufficiently small $\epsilon>0)$ ). If $\partial \Omega$ is (tangentially) graph-like on $D(p, r)$ for all $p \in \partial \Omega$, one says that $\partial \Omega$ is (tangentially) graph-like for radius $r$.

It is instructive to consider what is not considered graphlike or tangentially graph-like. Violations of the graph-like condition are generally due to a radius that is too large (certainly, choosing a radius so large that all of $\Omega$ is in the disk will do it). For example, a unit side length square is not graphlike with radius $(1 / 2)+\epsilon$ for any $\epsilon>0$ (position the circle at the center of a side; see Figure 3(a)). Notice that the same square is graph-like with any radius $1 / 2$ or below. A shape can fail to be tangentially graph-like while still being graph-like if it fails to be a graph in the required orientation but works in some other (see Figure 3(b)).

We would like to consider shapes with corners but our tangentially graph-like condition requires that the boundary be differentiable everywhere. The following definitions allow us to generalize the tangentially graph-like condition to this situation by using one-sided derivatives.

Definition 6. Given a piecewise $C^{1}$ function $\gamma:[0, L] \rightarrow \mathbb{R}^{2}$, one defines the tangent cone of $\gamma$ at a point $s$ (which is denoted by $\left.T_{\gamma}(s)\right)$ in terms of the one-sided derivatives. In particular, one lets $T_{\gamma}(s)=\left\{\alpha \Gamma^{-}+\beta \Gamma^{+} \mid \alpha, \beta \geq 0, \alpha+\beta>0\right\}$ where $\Gamma^{-}=\lim _{t \uparrow s} \gamma^{\prime}(t)$ and $\Gamma^{+}=\lim _{t \downarrow s} \gamma^{\prime}(t)$.

Definition 7. One extends the tangentially graph-like notion to boundaries that are piecewise $C^{1}$ by defining $\partial \Omega$ to be tangent-cone graph-like (TCGL) at a point $\gamma(s) \in \partial \Omega$ if it is graph-like at $\gamma(s)$ for every orientation in the tangent cone of $\partial \Omega$ at $s$. More precisely, for every $w \in T_{\gamma}(s)$ and every pair of distinct points $u, v \in \partial \Omega \cap D(p, r)$, one has $\langle w, u-v\rangle \neq 0$. See Figure 2(b).

Remark 8. It is clear that $T_{\gamma}(s)$ in Definition 6 is a convex cone. The tangent cone is dependent on the direction in which $\gamma$ traverses $\partial \Omega$ (which by convention was counterclockwise) since an arc-length traversal $\widehat{\gamma}(s, r)=\gamma(L-s, r)$ would have different tangent cones (namely, $w \in T_{\gamma}(s)$ iff $-w \in T_{\widehat{\gamma}}(s)$ ). However, these differences are irrelevant to the application of Definition 7.

Remark 9. Note that when $\partial \Omega$ is $C^{1}$, there is only one direction in $T_{\gamma}(s)$ for each $s$ (i.e., the tangent to $\partial \Omega$ at $\gamma(s))$. Thus, the definitions of tangentially graph-like and 


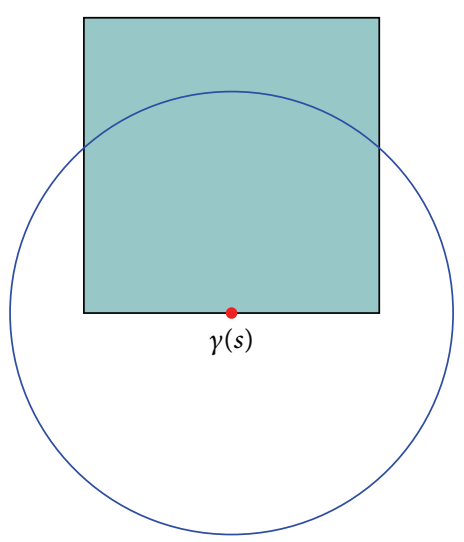

(a)

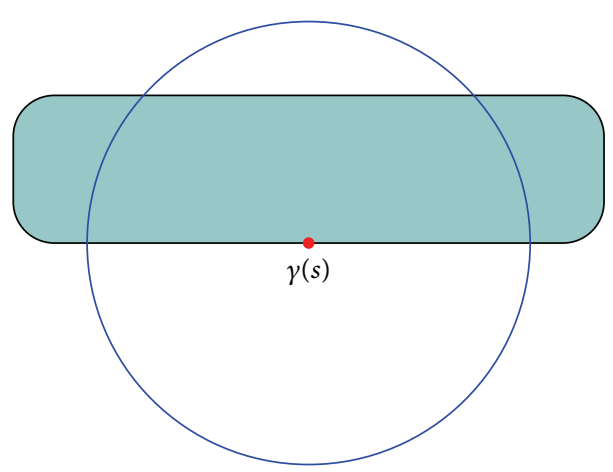

(b)

FIGURE 3: (a) The square is not graph-like with the indicated radius (no orientation makes it a graph). (b) The rounded rectangle is graph-like but not tangentially graph-like with the indicated center and radius.

tangent-cone graph-like coincide when $\partial \Omega$ is $C^{1}$ and every tangentially graph-like boundary is tangent-cone graph-like.

2.2. Two-Arc Property. The graph-like condition implies (in proof of the following lemma) that $\Omega$ will never be entirely contained in the disk, no matter where on the boundary we center it. That is, some part of $\Omega$ lies outside of $D(p, r)$ for every $p \in \partial \Omega$.

Lemma 10. Let $r \in \mathbb{R}^{+}$and $p \in \partial \Omega$. If $\partial \Omega$ is graph-like on $D(p, r)$, then $|\partial \Omega \cap C(p, r)| \geq 2$.

Proof. Suppose by way of contradiction that $|\partial \Omega \cap C(p, r)|<$ 2. Since $\partial \Omega$ is a simple closed curve, we have $\partial \Omega \subseteq D(p, r)$. As $\partial \Omega$ is graph-like at $p$ with radius $r$, there exists some orientation for which $\partial \Omega \cap D(p, r)=\partial \Omega$ is the graph of a well-defined function. However, $\partial \Omega$ is a simple closed curve so it is not the graph of a function in any orientation, yielding a contradiction.

The next result is the reason we find the tangent-cone graph-like condition useful. It says that if $\partial \Omega$ is tangent-cone graph-like with radius $r$, then, for every $p \in \partial \Omega$, the disk $D(p, r)$ has only two points of intersection with $\partial \Omega$ and these are transverse. In other words, this means that when working locally in the disk $D(p, r)$, we need only to consider a single piece of $\partial \Omega$.

Theorem 11. If $\partial \Omega$ is tangent-cone graph-like with radius $r \in$ $\mathbb{R}^{+}$at $p \in \partial \Omega$, then $|\partial \Omega \cap C(p, r)|=2$ and $\partial \Omega$ crosses $C(p, r)$ transversely at these points. As a result, for every $q_{1}, q_{2} \in$ $\partial \Omega \cap D(p, r)$, there is a unique arc along $\partial \Omega$ between them in $D(p, r)$.

Proof. By Lemma 10, we have that $|\partial \Omega \cap C(p, r)| \geq 2$. Note that $\partial \Omega$ contains an interior point $(p)$ and at least two boundary points of the disk $D(p, r)$ (since $|\partial \Omega \cap C(p, r)| \geq 2$ ). As $\partial \Omega$ is connected and simply closed, there must exist an arc of $\partial \Omega$ within the disk going from some point on $C(p, r)$ through $p$ to another point on $C(p, r)$.
Suppose $|\partial \Omega \cap C(p, r)|>2$; that is, there are other points of intersection. Letting $q$ denote one of these, there are two cases to consider (illustrated in Figure 4).

(a) $\partial \Omega$ Does Not Cross $C(p, r)$ at $q$. As $\partial \Omega$ is tangentcone graph-like at $q$, then $\partial \Omega \cap C(q, r)$ is a graph in every orientation in the tangent cone of $\partial \Omega$ at $q$. In particular, note that the tangent line to $C(p, r)$ at $q$ is in this cone. However, the line from $p$ to $q$ is normal to this line and thus $\partial \Omega \cap$ $C(q, r)$ is not graph-like in this orientation, a contradiction. Therefore, this case cannot occur. This argument applies to all points in $\partial \Omega \cap C(p, r)$ so we immediately have the result that $\partial \Omega$ always crosses $C(p, r)$ transversely.

(b) $\partial \Omega$ Crosses $C(p, r)$ at $q$. There exists $q^{\prime} \in \partial \Omega \cap C(p, r)$ such that there is a path along $\partial \Omega$ in $D(p, r)$ from $q$ to $q^{\prime}$. That is, there exist $s_{1}, s_{2} \in[0, L)$ (without loss of generality, $s_{1}<s_{2}$ ) such that $\gamma\left(s_{1}\right)=q, \gamma\left(s_{2}\right)=q^{\prime}$, and the image of $\left[s_{1}, s_{2}\right]$ under $\gamma$ is contained in $D(p, r)$ (but does not include $p$, since it is on another arc and $\partial \Omega$ is simple). Thus, $\gamma$ enters $C(p, r)$ at $s_{1}$ and exits at $s_{2}$.

If we can find $s \in\left[s_{1}, s_{2}\right]$ and $w$ in the tangent cone of $\partial \Omega$ at $\gamma(s)$ satisfying $\langle w, p-\gamma(s)\rangle=0$, we will contradict that $\partial \Omega$ is tangent-cone graph-like.

Define $v:\left[s_{1}, s_{2}\right] \rightarrow \mathbb{R}^{2}$ by

$$
v(s)= \begin{cases}\lim _{t \downarrow s_{1}} \gamma^{\prime}(s), & s=s_{1}, \\ \lim _{t \uparrow s} \gamma^{\prime}(s), & s \in\left(s_{1}, s_{2}\right] .\end{cases}
$$

Note that $v(s)$ is in the tangent cone of $\partial \Omega$ at $\gamma(s)$ so that $\partial \Omega \cap$ $D(\gamma(s), r)$ is graph-like using the orientation given by $v(s)$.

Define $\phi(s):\left[s_{1}, s_{2}\right] \rightarrow \mathbb{R}$ by $\phi(s)=\langle v(s), p-\gamma(s)\rangle$. Note that from $\gamma\left(s_{1}\right)$ both $v\left(s_{1}\right)$ and $p-\gamma\left(s_{1}\right)$ are directions pointing into the circle so $\phi\left(s_{1}\right)>0$. Similarly, $v\left(s_{2}\right)$ points out and $p-\gamma\left(s_{2}\right)$ points in so that $\phi\left(s_{2}\right)<0$.

Observe that $v$ (and therefore $\phi$ ) is piecewise continuous since $\gamma$ is piecewise $C^{1}$. By a piecewise continuous analogue of 

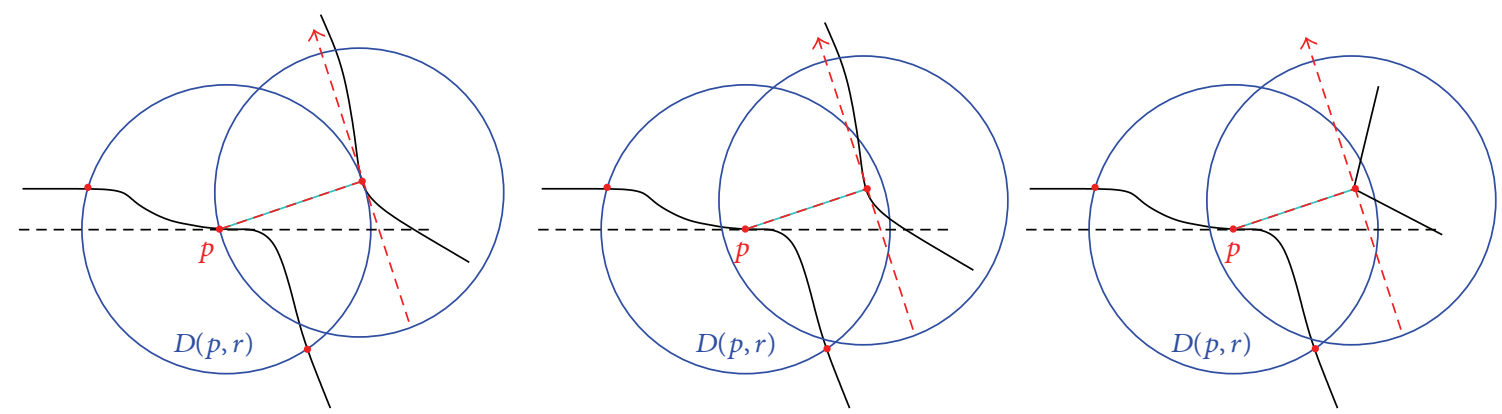

FIGURE 4: Additional points of intersection violate the TCGL condition.

the intermediate value theorem, there exists $\bar{s} \in\left[s_{1}, s_{2}\right]$ such that

$$
\lim _{t \rightarrow \bar{s}^{-}} \phi(t) \leq 0 \leq \lim _{t \rightarrow \bar{s}^{+}} \phi(t) .
$$

By continuity of the inner product and $\gamma$, we have

$$
\lim _{t \rightarrow \bar{s}^{-}} \phi(t)=\left\langle\lim _{t \rightarrow \bar{s}^{-}} \gamma^{\prime}(t), p-\gamma(\bar{s})\right\rangle .
$$

Similarly, $\lim _{t \rightarrow \bar{s}^{+}} \phi(t)=\left\langle\lim _{t \rightarrow \bar{s}^{+}} \gamma^{\prime}(t), p-\gamma(\bar{s})\right\rangle$.

If $\gamma$ is differentiable at $\bar{s}$, then $\phi(\bar{s})=\lim _{t \rightarrow \bar{s}} \phi(t)=0$ and we have our contradiction. Otherwise, let $w_{1}=\lim _{t \rightarrow \bar{s}^{-}} \gamma^{\prime}(t)$ and $w_{2}=\lim _{t \rightarrow \bar{s}^{+}} \gamma^{\prime}(t)$. As both $w_{1}$ and $w_{2}$ are in the convex tangent cone of $\partial \Omega$ at $\gamma(\bar{s})$, any positive linear combination of them is as well. Letting $\psi(\lambda)=\lambda w_{1}+(1-\lambda) w_{2}$, we have

$$
\langle\psi(0), p-\gamma(\bar{s})\rangle \leq 0 \leq\langle\psi(1), p-\gamma(\bar{s})\rangle .
$$

Noting that $\psi$ is continuous in $\lambda$, we apply the intermediate value theorem to obtain $\bar{\lambda} \in(0,1)$ such that $\langle\psi(\bar{\lambda}), p-\gamma(\bar{s})\rangle=$ 0 . Letting $w=\psi(\bar{\lambda})$, we obtain our contradiction.

Therefore, there are no other points of intersection and $|\partial \Omega \cap C(p, r)|=2$.

Definition 12. One says that $\Omega$ has the two-arc property for a given radius $r$ if, for every point $p \in \partial \Omega$, one has that $D(p, r)$ divides $\partial \Omega$ into two connected arcs: $\partial \Omega \cap D(p, r)$ and $\partial \Omega \backslash D(p, r)$. Instead of considering how $D(p, r)$ divides $\partial \Omega$, one can equivalently frame the definition in terms of how $\partial \Omega$ divides $C(p, r)$. That is, $\Omega$ has the two-arc property if the circle $C(p, r)$ is divided into two connected arcs by $\partial \Omega$ for every $p \in \partial \Omega$.

Corollary 13. If $\Omega$ is tangent-cone graph-like for some radius $r$, then it has the two-arc property.

Proof. This is a trivial consequence of Theorem 11.

Corollary 14. If $\Omega$ is tangentially graph-like for some radius $r$, then it has the two-arc property for radius $r$.

Remark 15. While the assumption of the two-arc property for disks of radius $r=\widehat{r}$ does not imply the two-arc property for all $r<\widehat{r}$ (see Figure 5), it is the case that TGL for $r=\widehat{r}$ does imply that $\gamma$ is TGL for all $0<r<\widehat{r}$. The fact that $\gamma$ is TGL for all $0<r<\widehat{r}$ follows easily from the definition of TGL and the fact that $D(p, r) \subsetneq D(p, \widehat{r})$.

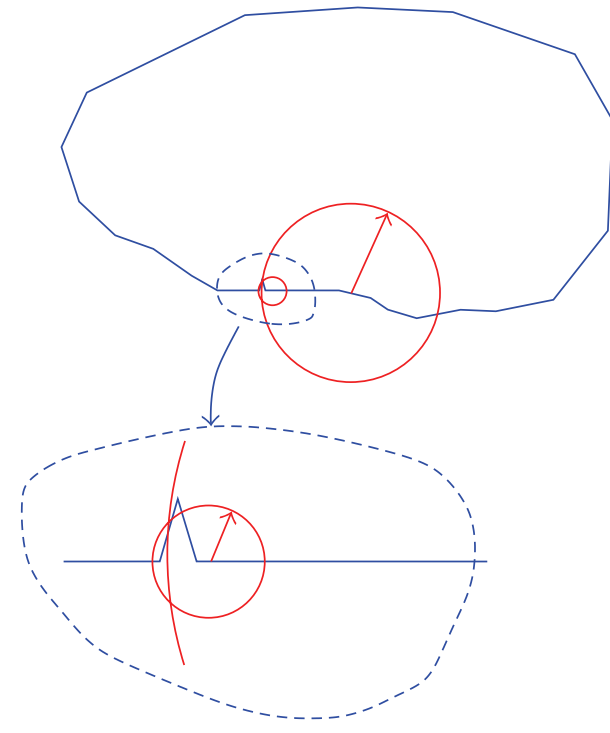

FIGURE 5: The two-arc property for $r=\widehat{r}$ does not imply that it holds for all $r<\widehat{r}$.

2.3. Notation. Suppose that $\partial \Omega$ is tangent-cone graph-like with radius $r$ and we have some $s \in[0, L)$ such that $\partial \Omega$ is tangentially graph-like at $\gamma(s)$ with radius $r$. Since $\partial \Omega$ is TGL at $\gamma(s)$, it has two points of intersection with $C(\gamma(s), r)$ by Theorem 11. In the orientation forced by the TGL condition, one of these points of intersection must be on the right side of the circle and one must be on the left side.

With reference to Figure 6 we define $s^{+}(s)$ and $s^{-}(s) \epsilon$ $[0, L)$ so that $\gamma\left(s^{+}(s)\right)$ is the point of intersection on the right and $\gamma\left(s^{-}(s)\right)$ is the point of intersection on the left. The notation is motivated by the fact that $0<s^{-}(s)<s<$ $s^{+}(s)<L$ in general due to our convention that $\gamma$ traverses $\partial \Omega$ counterclockwise. The only case where this is not true is when $\gamma(L)=\gamma(0)$ is in the disk but even then it will hold for a suitably shifted $\hat{\gamma}$ that starts at some point outside the current disk.

The quantities $\theta_{1}(s)$ and $\theta_{2}(s)$ are the angles that the rays from the origin to the right and left points of intersection, respectively, make with the positive $x$ axis. We can assume $\theta_{1}(s) \in(-\pi / 2, \pi / 2)$ and $\theta_{2}(s) \in(\pi / 2,3 \pi / 2)$.

We define $\nu_{1}(s)$ as the angle between the vector $\gamma\left(s^{+}(s)\right)-$ $\gamma(s)$ and the vector $\lim _{t \downarrow s^{+}(s)} \gamma^{\prime}(t)$, the one-sided tangent to $\partial \Omega$ at the point of intersection on the right. 


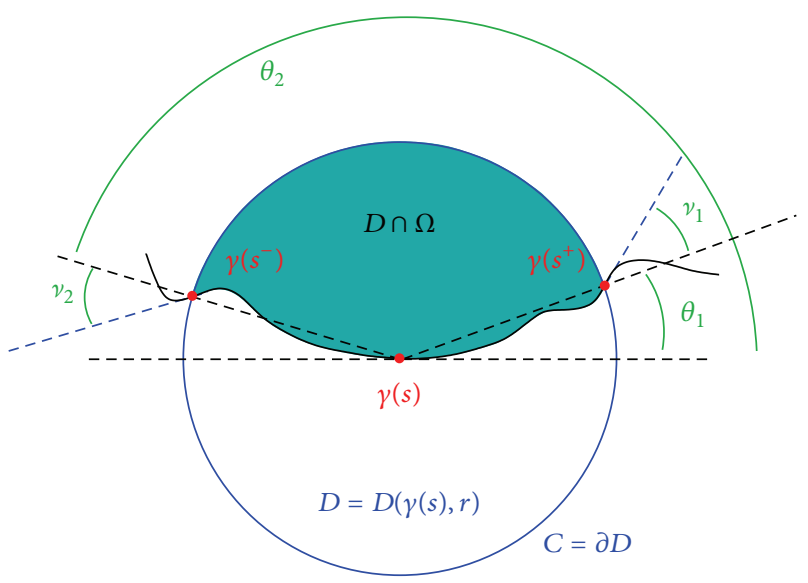

FIGURE 6: Notation and conventions.

That is, we are measuring the angle between the outward normal to the disk at the point of intersection and the actual direction $\gamma$ is going as it exits the disk. We define $v_{2}(s)$ similarly. We have $\nu_{1}, \nu_{2} \in(-\pi / 2, \pi / 2)$ due to the fact that all circle crossings are transverse by Theorem 11 .

When the proper $s$ to use is implied by context, we will often simply write $s^{+}, s^{-}, \theta_{1}, \theta_{2}, v_{1}$, and $v_{2}$ in place of $s^{+}(s), s^{-}(s)$, and so forth.

2.4. Calculus on Tangent Cones. The following result is a version of the intermediate value theorem for elements of the tangent cones.

Lemma 16. Suppose that $\partial \Omega$ is tangent-cone graph-like on $D(\gamma(s), r)$ and $s_{1}<s_{2}$ such that $\gamma\left(s_{1}\right), \gamma\left(s_{2}\right) \in D(\gamma(s), r)$. Further suppose that $w_{1} \in T_{\gamma}\left(s_{1}\right), w_{2} \in T_{\gamma}\left(s_{2}\right)$, and $\alpha \in(0,1)$, and let $w^{\prime}=\alpha w_{1}+(1-\alpha) w_{2}$. Then, there exists $s^{\prime} \in\left[s_{1}, s_{2}\right]$ such that either $w^{\prime}$ or $-w^{\prime}$ is in $T_{\gamma}\left(s^{\prime}\right)$.

Proof. Let $n$ be a unit vector in $\mathbb{R}^{2}$ with $n \perp\left(\alpha w_{1}+(1-\alpha) w_{2}\right)$. We have $\alpha\left\langle n, w_{1}\right\rangle=-(1-\alpha)\left\langle n, w_{2}\right\rangle$. It suffices to consider only $\left\langle n, w_{1}\right\rangle \leq 0 \leq\left\langle n, w_{2}\right\rangle$ as the argument is identical in the other case. Note that since $0 \leq\left\langle n, w_{2}\right\rangle=c_{1}\left\langle n, \lim _{t \uparrow s_{2}} \gamma^{\prime}(t)\right\rangle+$ $c_{2}\left\langle n, \lim _{t \downarrow s_{2}} \gamma^{\prime}(t)\right\rangle$ for some nonnegative constants $c_{1}, c_{2}$ not both zero, at least one of the inner products on the right is nonnegative. Using the notation of Definition 6, we define $M_{2}=\arg \max _{\Gamma \in\left\{\Gamma^{+}, \Gamma^{-}\right\}}\langle n, \Gamma\rangle$ and have $\left\langle n, M_{2}\right\rangle \geq 0$. We similarly define $M_{1}$ with respect to $w_{1}$ such that $\left\langle n, M_{1}\right\rangle \leq 0$.

Define

$$
v(t)=\left\{\begin{array}{l}
M_{i}, \\
\lim _{t \uparrow t} \gamma^{\prime}(s)
\end{array} \quad t=s_{i}, \quad i=1,2\right.
$$

and $\phi(t)=\langle n, v(t)\rangle$. Since $\phi\left(s_{1}\right) \leq 0 \leq \phi\left(s_{2}\right)$, the argument proceeds as in Theorem 11 to yield $\bar{s} \in\left[s_{1}, s_{2}\right]$ and $\bar{w} \in T_{\gamma}(\bar{s})$ such that $\langle n, \bar{w}\rangle=0$. Thus, $\bar{w}=k w^{\prime}$ for some $k \neq 0$. In particular, $w^{\prime}=(1 / k) \bar{w}$ so either $w^{\prime} \in T_{\gamma}(\bar{s})$ or $-w^{\prime} \in T_{\gamma}(\bar{s})$ (depending on the sign of $k$ ).
In addition to the intermediate value theorem, we have an analogous mean value theorem for tangent cone elements.

Lemma 17. Suppose that $\gamma:[a, b] \rightarrow \mathbb{R}^{2}$ is a simple, arc-length parameterized curve with piecewise continuous derivative defined on $(a, b)$ except possibly on finitely many points. Further suppose that the image of $\gamma$ has no cusps. Then there exists $c$ in $(a, b)$ such that either $\gamma(b)-\gamma(a)$ or $-(\gamma(b)-$ $\gamma(a))$ is in $T_{\gamma}(c)$.

Proof. Let $n$ be a unit vector with $\langle\gamma(b)-\gamma(a), n\rangle=0$. Consider $\psi(t)=\langle\gamma(t), n\rangle$ and note that $\psi^{\prime}(t)=\left\langle\gamma^{\prime}(t), n\right\rangle$ is defined wherever $\gamma(t)$ is differentiable. We have $\int_{a}^{b} \psi^{\prime}(t)=$ $\psi(b)-\psi(a)=\langle\gamma(b)-\gamma(a), n\rangle=0$. Thus, either $\psi^{\prime}(t)=$ 0 everywhere it is defined or it takes on both positive and negative values. In particular, there exists a point $c \in(a, b)$ such that either $\psi^{\prime}(c)=0$ or $\lim _{t \uparrow c} \psi^{\prime}(t) \leq 0 \leq \lim _{t \downarrow c} \psi^{\prime}(t)$.

If $\psi^{\prime}(c)=0$, then we have $\left\langle\gamma^{\prime}(c), n\right\rangle=0$ so that $\gamma^{\prime}(c)=$ $k(\phi(b)-\phi(a))$ for some $k \neq 0$. As $\gamma^{\prime}(c) \in T_{\gamma}(c)$, we have $(k /|k|)(\phi(b)-\phi(a)) \in T_{\gamma}(c)$ which gives us our conclusion.

If $\lim _{t \uparrow c} \psi^{\prime}(t) \leq 0 \leq \lim _{t \downarrow c} \psi^{\prime}(t)$, there exists $\alpha \in(0,1)$ such that $0=\alpha \lim _{t \uparrow c} \psi^{\prime}(t)+(1-\alpha) \lim _{t \downarrow c} \psi^{\prime}(t)$. Note that $\lim _{t \uparrow c} \psi^{\prime}(t)=\left\langle w_{1}, n\right\rangle$ and $\lim _{t \downarrow c} \psi^{\prime}(t)=\left\langle w_{2}, n\right\rangle$ for some $w_{1}, w_{2} \in T_{\gamma}(c)$ and let $w^{\prime}=\alpha w_{1}+(1-\alpha) w_{2}$.

By the convexity of $T_{\gamma}(c)$, we have $w^{\prime} \in T_{\gamma}(c)$ with $\left\langle w^{\prime}, n\right\rangle=0$ which follows as in the previous case.

The following lemma tells us that the tangent-cone graphlike condition is sufficient to apply Lemma 17.

Lemma 18. If $\partial \Omega$ is tangent-cone graph-like for some radius $r$, then $\partial \Omega$ has no cusps.

Proof. Suppose $\partial \Omega$ has a cusp at $\gamma(s)$. Then, using the terminology of Definition 6 and the fact that $\gamma$ is arc length parameterized, we have $\Gamma^{+}=-\Gamma^{-}$. We let $w=0$ and note that $w=\Gamma^{+}+\Gamma^{-} \in T_{\gamma}(s)$. Letting $u, v \in \partial \Omega \cap D(\gamma(s), r)$ with $u \neq v$, we have $\langle w, u-v\rangle=0$, contradicting the fact that $\partial \Omega$ is tangent-cone graph-like. Therefore, $\partial \Omega$ has no cusps.

2.5. TCGL Boundary Properties. The following technical lemmas allow us to bound various distances and areas encountered in tangent-cone graph-like boundaries.

Lemma 19. Suppose that $\partial \Omega$ is tangent-cone graph-like with radius $r$ and points $p_{1}, p_{2} \in \partial \Omega$ with $d\left(p_{1}, p_{2}\right)<r$. Then one of the arcs (call it $P$ ) along $\partial \Omega$ between $p_{1}$ and $p_{2}$ is such that, for any two points $q_{1}, q_{2} \in P$, one has $d\left(q_{1}, q_{2}\right)<r$.

Proof. Note that $p_{2} \in D\left(p_{1}, r\right)$ so that there is an arc along $\partial \Omega$ from $p_{1}$ to $p_{2}$ which is fully contained in the interior of $D\left(p_{1}, r\right)$ by Theorem 11 . We will call this $\operatorname{arc} P$.

For all $x$ on $P$, let $P_{x}$ denote the subpath of $P$ from $p_{1}$ to $x$ (so $P=P_{p_{2}}$ ). We claim that $P_{x}$ is contained in $D(x, r)$ for all $x$ on $P$ (thus, $P$ is contained in $D\left(p_{2}, r\right)$ ). Indeed, if this were not the case, then there must be some $\widehat{x}$ on $P$ such that $P_{\widehat{x}}$ is contained in $D(\widehat{x}, r)$ but $C(\widehat{x}, r) \cap P_{\widehat{x}}$ is nonempty (i.e., we can 
move the disk along $P$ until some part of the subpath hits the boundary). That is, the subpath $P_{\widehat{x}}$ has a tangency with the disk $D(\widehat{x}, r)$ which is impossible because of Theorem 11.

Let $q_{1} \in P$ and note that since $P_{x}$ is contained in $D(x, r)$ for all $x$ on $P$, we have that $P$ is contained in $D\left(q_{1}, r\right)$. Therefore, $d\left(q_{1}, q_{2}\right)<r$ for all $q_{1}, q_{2} \in P$ as desired.

Lemma 20. If $q_{1}=\gamma\left(s_{1}\right), q_{2}=\gamma\left(s_{2}\right) \in P$ where $P$ is as in the previous lemma, then the arc length between $q_{1}$ and $q_{2}$ along $P$ is at most $\sqrt{2} d\left(q_{1}, q_{2}\right)$.

Proof. Since $\Omega$ is tangentially graph-like, for any $w_{1} \in T_{\gamma}\left(s_{1}\right)$, $w_{2} \in T_{\gamma}\left(s_{2}\right)$, the angle between $w_{1}$ and $w_{2}$ is at most $\pi / 2$. Since this is true for all $q \in P$, there is a point $q^{\prime}=\gamma\left(s^{\prime}\right) \in P$ and $w^{\prime} \in T_{\gamma}\left(s^{\prime}\right)$ such that the angle between $w^{\prime}$ and tangent vectors for any other point $q \in P$ is at most $\pi / 4$.

This means that $P$ is the graph of a Lipschitz function $g$ of rank 1 in the orientation defined by $w^{\prime}$. This does not necessarily imply that $D\left(q^{\prime}, r\right) \cap \partial \Omega, D\left(p_{1}, r\right) \cap \partial \Omega$, or $D\left(p_{2}, r\right) \cap \partial \Omega$ is the graph of a Lipschitz function; we explore a Lipschitz condition for the disks in Section 3. Let $x_{1}, x_{2} \in$ $[-r, r]$ with $p_{1}=\left(x_{1}, g\left(x_{1}\right)\right), p_{2}=\left(x_{2}, g\left(x_{2}\right)\right)$. Then the arclength from $p_{1}$ to $p_{2}$ is given by

$$
\begin{aligned}
& \int_{x_{1}}^{x_{2}} \sqrt{1+g^{\prime}(x)^{2}} d x \\
& \quad \leq \int_{x_{1}}^{x_{2}} \sqrt{2} d x=\sqrt{2}\left(x_{2}-x_{1}\right) \leq \sqrt{2} d\left(p_{1}, p_{2}\right) .
\end{aligned}
$$

Lemma 21. If $\gamma$ is tangent-cone graph-like with radius $r$ and $0 \leq s_{1} \leq s_{2}<L$ with $d\left(\gamma\left(s_{1}\right), \gamma\left(s_{2}\right)\right)=\delta<r$, then the image of $\left[s_{1}, s_{2}\right]$ together with the straight line from $\gamma\left(s_{1}\right)$ to $\gamma\left(s_{2}\right)$ encloses a region with $O\left(\delta^{2}\right)$ area.

Proof. By Lemma 20, we have that the image of $\left[s_{1}, s_{2}\right]$ under $\gamma$ has arc length $s_{2}-s_{1} \leq \sqrt{2} \delta$. Therefore, the region of interest has perimeter at most $(\sqrt{2}+1) \delta$ so by the isoperimetric inequality it has area at most $\left((\sqrt{2}+1)^{2} / 4 \pi\right) \delta^{2}$ from which the conclusion follows.

\section{TCGL Polygonal Approximations}

If $\Omega$ is tangent-cone graph-like with radius $r$, it can sometimes be nice to know that there is an approximating polygon to $\Omega$ which is also tangent-cone graph-like. The following lemmas explore this idea.

Lemma 22. If $\partial \Omega$ is TCGL with radius $r$, then, for each $\epsilon \epsilon$ $(0, r)$, there exists a polygonal approximation to $\partial \Omega$ that is TCGL with radius $r-\epsilon$ and such that every point on $\partial \Omega$ is within distance $\epsilon / 6$ of the polygon.

Proof. First, choose a finite number of points along the boundary such that the arc length along $\gamma$ between any two neighboring points is no more than $\epsilon / 3$. These will be the vertices of our polygon. Similarly to $\gamma$, we let $\phi$ be an arclength parameterization of this polygon so that they both encounter their common points in the same order.
The fine spacing between vertices guarantees that we obtain the $\epsilon / 6$ bound. Indeed, given any point $p \in \partial \Omega$ and its neighboring vertices $v_{1}$ and $v_{2}$, the arc length along $\partial \Omega$ from $v_{1}$ to $p$ plus that from $p$ to $v_{2}$ is at most $\epsilon / 3$ by assumption. Since Euclidean distance is bounded above by arc length, we have $d\left(p, v_{1}\right)+d\left(p, v_{2}\right) \leq \epsilon / 3$. This bound in turn implies that at least one of $d\left(p, v_{1}\right)$ and $d\left(p, v_{2}\right)$ is bounded above by $\epsilon / 6$.

Consider a point $p=\phi(t)$ on a side of the polygon (i.e., not a vertex) and its neighboring vertices $v_{1}=\phi\left(t_{1}\right)=\gamma\left(s_{1}\right)$ and $v_{2}=\phi\left(t_{2}\right)=\gamma\left(s_{2}\right)$ (chosen with $t_{1}<t<t_{2}$ and $s_{1}<s_{2}$ ). By Lemma 17, there exists $s \in\left(s_{1}, s_{2}\right)$ such that $v_{2}-v_{1} \in T_{\gamma}(s)$. Note that this is the only member of $T_{\phi}(t)$ up to positive scalar multiplication.

Combining the arcs along $\gamma$ and $\phi$ between $v_{1}$ and $v_{2}$, we obtain a closed curve with total length at most $2 \epsilon / 3$, so that the distance between any two points on the curve is at most $\epsilon / 3$. That is, for any $s^{\prime} \in\left[s_{1}, s_{2}\right]$ and $t^{\prime} \in\left[t_{1}, t_{2}\right]$, we have $d\left(\gamma\left(s^{\prime}\right), \phi\left(t^{\prime}\right)\right) \leq \epsilon / 3$.

Let $x \in D(\phi(t), r-\epsilon)$. Then $d(x, \gamma(s)) \leq d(x, \phi(t))+$ $d(\phi(t), \gamma(s)) \leq r-(2 \epsilon / 3)$ so that $D(\phi(t), r-\epsilon)$ is contained in $D(\gamma(s), r-(2 \epsilon / 3))$.

Let $a, b$ be distinct points on the polygon in $D(\phi(t), r-$ $\epsilon)$ and consider the line connecting them. This line also intersects $a^{\prime}, b^{\prime}$ on $\gamma$ such that we have $a^{\prime} \neq b^{\prime}, d\left(a, a^{\prime}\right) \leq \epsilon / 3$, and $d\left(b, b^{\prime}\right) \leq \epsilon / 3$ so that $a^{\prime}, b^{\prime} \in \partial \Omega \cap D(\gamma(s), r)$. As $a-b=$ $c\left(a^{\prime}-b^{\prime}\right)$ for some scalar $c>0$, we have

$$
\left\langle v_{2}-v_{1}, a-b\right\rangle=c\left\langle v_{2}-v_{1}, a^{\prime}-b^{\prime}\right\rangle \neq 0,
$$

since $\gamma$ is TCGL at $\gamma(s)$ with radius $r$ and $v_{2}-v_{1} \in T_{\gamma}(s)$. Thus, $\phi$ is TCGL at $p$ with radius $r-\epsilon$.

The case where $p=\phi(t)$ is a vertex is similar but we must consider an arbitrary vector $w \in T_{\phi}(t)$ in the inner product. We wish to show that, for every $w \in T_{\phi}(t)$, there is a $s^{\prime}$ such that either $w$ or $-w \in T_{\gamma}\left(s^{\prime}\right)$ and $d\left(p, \gamma\left(s^{\prime}\right)\right) \leq \epsilon / 3$, after which the proof follows as in the first case with $w$ (or $-w$ ) in place of $v_{2}-v_{1}$. We let $\gamma(s)=\phi(t)=p$ and let $v_{1}=\phi\left(t_{1}\right)=\gamma\left(s_{1}\right)$ and $v_{2}=\phi\left(t_{2}\right)=\gamma\left(s_{2}\right)$ be the neighboring vertices (so $t_{1}<t<t_{2}$ and $s_{1}<s<s_{2}$ ).

As above, there exist $s_{1}^{\prime}, s_{2}^{\prime}$ such that $s_{1} \leq s_{1}^{\prime} \leq s \leq s_{2}^{\prime} \leq s_{2}$, $\gamma(s)-\gamma\left(s_{1}\right) \in T_{\gamma}\left(s_{1}^{\prime}\right)$, and $\gamma\left(s_{2}\right)-\gamma(s) \in T_{\gamma}\left(s_{2}^{\prime}\right)$. Note that $T_{\phi}(t)$ is exactly the set of positive linear combinations of these vectors. By Lemma 16, for every $w \in T_{\phi}(t)$, there is a $s^{\prime} \epsilon$ $\left[s_{1}^{\prime}, s_{2}^{\prime}\right]$ such that $w \in T_{\gamma}\left(s^{\prime}\right)$. As $d\left(p, \gamma\left(s^{\prime}\right)\right)<\epsilon / 3$, the proof is complete.

Definition 23. One says that $\Omega$ is tangentially graph-like and Lipschitz (TGLL) with radius $r$ if $\Omega$ is tangentially graphlike with radius $r$ and there is some constant $0<K<\infty$ such that, for every $p \in \partial \Omega$, the $\operatorname{arc} D(p, r) \cap \partial \Omega$ is the graph of a Lipschitz function (in the same orientation used by the tangentially graph-like definition) and that the Lipschitz constant is at most $K$. 
Remark 24. Note that tangentially graph-like does not imply tangentially graph-like and Lipschitz: taking $\gamma$ to be a square with side length 5 whose corners are replaced by quarter circles of radius 1 and then considering disks of radius $\sqrt{2}$ centered on $\gamma$ yields one example.

Because $\gamma$ is arclength parameterized by $s,\left\|\gamma^{\prime}(s)\right\|=1$ for all $s$. Since $\gamma$ is assumed $C^{1}$ on its compact domain $[0, L], \gamma^{\prime}$ is uniformly continuous: for any $\epsilon>0$, there is a $\delta_{\epsilon}$ such that if $\left|s_{2}-s_{1}\right|<\delta_{\epsilon}$, then $\left\|\gamma^{\prime}\left(s_{2}\right)-\gamma^{\prime}\left(s_{1}\right)\right\|<\epsilon$.

We will use the fact that $\gamma$ always crosses $\partial D$ transversely to prove that $\gamma$ is in fact TGLL on slightly bigger disks of radius $r+\delta$ as long as one takes a somewhat bigger Lipschitz constant $\widehat{K}$. It is then an immediate result of Lemma 22 that we can find an approximating polygon that is TCGL with radius $r$.

Lemma 25. If $\gamma$ is TGLL with radius $r$, then it is TGLL with radius $r+\delta$ for some $\delta>0$ and there is an approximating polygon $P_{\gamma}$ which is TCGL with radius $r$.

Proof. Step 1. Show that the quantities $v_{1}$ and $v_{2}$ are continuous as a function of $s \in[0, L]$ (see Figure 6).

Define $R^{2}(s, t) \equiv\|\gamma(s)-\gamma(t)\|^{2}$. Taking the derivative, we get

$$
D R=\left[\left\langle\frac{\gamma(s)-\gamma(t)}{R(s, t)}, \gamma^{\prime}(s)\right\rangle,\left\langle\frac{\gamma(t)-\gamma(s)}{R(s, t)}, \gamma^{\prime}(t)\right\rangle\right] .
$$

Because $\nu_{1}$ and $\nu_{2}$ are both less than $\pi / 2$ and $\gamma$ is graph-like in the disk, we have that both elements of this derivative are nowhere zero. By the implicit function theorem, we get that $s^{-}(s)$ and $s^{+}(s)$ are continuous functions of $s$. From this it follows that $v_{1}$ and $v_{2}$ are continuous on $[0, L]$.

Step 2. From the previous step and the compactness of $[0, L]$, we get that $\nu_{1}(s)$ and $\nu_{2}(s)$ are both bounded by $M_{v}<\pi / 2$. We define $\epsilon_{v} \equiv \pi / 2-M_{v}>0$. Fix a $t \in[0, L]$. Define $\hat{\rho}(s)$ by $\hat{\rho}^{2}(s)=R^{2}(s, t)=\|\gamma(s)-\gamma(t)\|^{2}$. Then $\dot{\hat{\rho}}(s)=$ $\left\langle(\gamma(s)-\gamma(t)) / \hat{\rho}, \gamma^{\prime}(s)\right\rangle=\left\langle n_{t}(s), \gamma^{\prime}(s)\right\rangle$ where $n_{t}(s)=(\gamma(s)-$ $\gamma(t)) /\|\gamma(s)-\gamma(t)\|=(\gamma(s)-\gamma(t)) / \hat{\rho}$, the external normal to $\partial D(\gamma(t), \hat{\rho})$ at $\gamma(s)$ (see Figure 7). On any interval in $s$ where $\dot{\hat{\rho}}(s)>0$ we have that $\hat{\rho}(s)$ is one to one and strictly increasing. Define $s^{*} \equiv s^{+}(t)$ and $s_{*} \equiv s^{-}(t)$. We showed above that $\dot{\hat{\rho}}\left(s^{*}\right)=\left\langle n_{t}\left(s^{*}\right), \gamma^{\prime}\left(s^{*}\right)\right\rangle \geq \cos \left(M_{\nu}\right)>0$.

For $\left\langle n_{t}(s), \gamma^{\prime}(s)\right\rangle=0, n_{t}(s)$ and $\gamma^{\prime}$ will have to have together turned by at least $\pi / 2-M_{\nu}$ radians. And until they have turned this far, $\left\langle n_{t}(s), \gamma^{\prime}(s)\right\rangle>0$. But $\dot{n}_{t}(s) \leq 1 / \rho \leq$ $1 / r_{\min }$ for some $r_{\min }>0$. (Choosing $r_{\min }=r / 2$ works.) And $\gamma^{\prime}$ is uniformly continuous on $[0, L]$. Therefore, there is a $\delta_{s}$ such that on $\left[s^{*}, s^{*}+\delta_{s}\right], n_{t}(s)$, and $\gamma^{\prime}$ both turn by less than $\epsilon_{\gamma} / 3$. Therefore, for $s \in\left[s^{*}, s^{*}+\delta_{s}\right]$, we have that $\left\langle n_{t}(s), \gamma^{\prime}(s)\right\rangle>\cos \left(\pi / 2-\epsilon_{\gamma} / 3\right)$ and $\gamma\left(\left[s^{*}, s^{*}+\delta_{s}\right)\right)$ intersects $C=\partial D(\gamma(t), \rho)$ once for each $\rho \in\left[r, r+\delta_{r}\right]$, where $\delta_{r} \equiv$ $\delta_{s} \cos \left(\pi / 2-\epsilon_{\nu} / 3\right)$.

A completely analogous argument works to show that $\gamma\left(\left[s_{*}-\delta_{s}, s_{*}\right]\right)$ intersects $C=\partial D(\gamma(t), \rho)$ once for each $\rho \in\left[r, r+\delta_{r}\right]$.

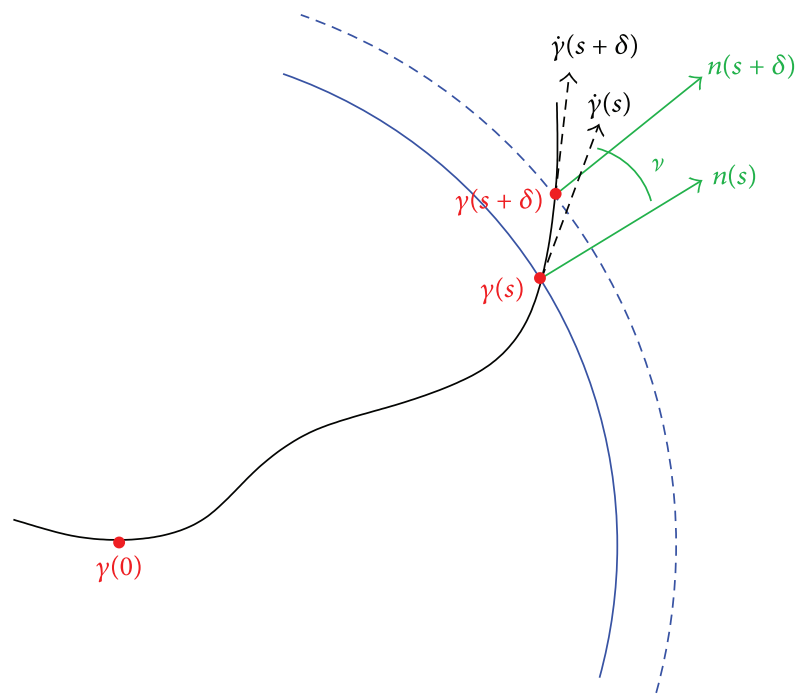

Figure 7: TGLL implies TCGL: notation.

Define $d(t)$ to be the distance from $D(\gamma(t), r)$ to $\gamma \backslash \gamma\left(\left[s_{*}-\right.\right.$ $\left.\left.\delta_{s}, s^{*}+\delta_{s}\right]\right)$. Since $\gamma$ is TGL, $d(t)$ is greater than zero for all $t$ and is continuous in $t$. Therefore, there is a smallest distance $\delta_{d}$ such that $d(t) \geq \delta_{d}$ for all $t$. Define $\delta_{\gamma_{o}}=\min \left(\delta_{d} / 2, \delta_{r} / 2\right)$.

Therefore, $\partial D(\gamma(t), \rho)$ intersects $\gamma$ exactly twice for $\rho \in$ $\left[r, r+\delta_{\gamma_{o}}\right]$ for any $t \in[0, L]$.

A similar argument shows that $\partial D(\gamma(t), \rho)$ intersects $\gamma$ exactly twice for $\rho \in\left[r-\delta_{\gamma_{i}}, r\right]$ for any $t \in[0, L]$. Defining $\delta_{\gamma} \equiv \min \left(\delta_{\gamma_{i}}, \delta_{\gamma_{o}}\right)$, we get that $\partial D(\gamma(t), \rho)$ intersects $\gamma$ exactly twice for $\rho \in\left[r-\delta_{\gamma}, r+\delta_{\gamma}\right]$, with the additional fact that $\left\langle n_{t}(s), \gamma^{\prime}(s)\right\rangle>\cos \left(\pi / 2-\epsilon_{\gamma} / 3\right)$ at all those intersections.

Step 3. TGLL implies that there is a constant $K<\infty$ such that $\gamma \cap D(\gamma(t), r)$ is the graph of a function whose $x$-axis direction is parallel to $\gamma^{\prime}(t)$ and this function is Lipschitz with Lipschitz constant $K$.

Since $\gamma^{\prime}$ is uniformly continuous, there will be a $\delta_{1}$ such that if $|u-v|<\delta_{1}$, then $\angle\left(\gamma^{\prime}(u), \gamma^{\prime}(v)\right)<\arctan 2 K-$ $\arctan K$. Define $\delta_{K, s}=\min \left(\delta_{s}, \delta_{1}\right)$. Define $\delta_{K, r}=$ $\min \left(\delta_{\gamma}, \delta_{K, s} \cos \left(\pi / 2-\epsilon_{\nu} / 3\right)\right)$. Then $\gamma \cap D\left(\gamma(t), r+\delta_{K, r}\right)$ is the graph of a Lipschitz function with Lipschitz constant at most $2 K$ when $\gamma^{\prime}(t)$ is used as the $x$-axis direction. That is, for all $t, \gamma$ is TGLL with Lipschitz constant $2 K$ for disks of radius $r+\delta_{K, r}$. The result follows by Lemma 22 .

\section{Derivatives of $g(s, r)$}

Lemma 26. Using the notation of Figure 6, we have $(\partial / \partial r) g(s, r)=\left(\theta_{2}-\theta_{1}\right) r$. That is, the derivative exists and equals the length of the curve $C(\gamma(s), r) \cap \Omega$.

Proof. We have the following (see Figure 8):

$$
\begin{aligned}
& \frac{\partial}{\partial r} g(s, r) \\
& =\lim _{\Delta r \rightarrow 0} \frac{\operatorname{Area}(\Omega \cap D(\gamma(s), r+\Delta r))-\operatorname{Area}(\Omega \cap D(\gamma(s), r))}{\Delta r} .
\end{aligned}
$$


This difference of areas can be modeled by the difference in the circular sectors of $D(\gamma(s), r+\Delta r)$ and $D(\gamma(s), r)$ with angle $\theta_{1}-\theta_{2}$. The actual area depends on the image of $\gamma$ outside of $D(\gamma(s), r)$, but this correction will be a subset of the circular segment of $D(\gamma(s), r+\Delta r)$ which is tangent to $D(\gamma(s), r)$ at the point $\gamma$ exits. This has area $O\left(\Delta r^{2}\right)$ by Lemma 21 .

Thus we have

$$
\begin{aligned}
\frac{\partial}{\partial r} & g(s, r) \\
& =\lim _{\Delta r \rightarrow 0} \frac{\left(\theta_{1}-\theta_{2}\right) r \Delta r+(1 / 2)\left(\theta_{1}-\theta_{2}\right) \Delta r^{2}+O\left(\Delta r^{2}\right)}{\Delta r} \\
& =\left(\theta_{1}-\theta_{2}\right) r .
\end{aligned}
$$

Lemma 27. Using the notation of Figures 6 and 9, one has $(\partial / \partial s) g(s, r)=h_{2}-h_{1}=r \sin \left(\theta_{2}\right)-r \sin \left(\theta_{1}\right)$.

Proof. We have

$$
\begin{aligned}
& \frac{\partial}{\partial s} g(s, r) \\
& =\lim _{\Delta s \rightarrow 0} \frac{\operatorname{Area}(\Omega \cap D(\gamma(s+\Delta s), r))-\operatorname{Area}(\Omega \cap D(\gamma(s), r))}{\Delta s} .
\end{aligned}
$$

The situation is illustrated in Figure 9 where we can see that the area being added as we go from $s$ to $s+\Delta s$ is the shaded region on the right with height $r-h_{1}$ and, considering firstorder terms only, uniform width $\Delta s$ so has area $\left(r-h_{1}\right) \Delta s$. Similarly, we are subtracting the area $\left(r-h_{2}\right) \Delta s$ on the left. Therefore, we have

$$
\frac{\partial}{\partial s} g(s, r)=\lim _{\Delta s \rightarrow 0} \frac{\left(r-h_{1}\right) \Delta s-\left(r-h_{2}\right) \Delta s}{\Delta s}=h_{2}-h_{1} .
$$

\section{Reconstructing Shapes from T-Like Data}

In this section, we consider the case where nonasymptotic densities and first derivatives are known along a T-shaped set (i.e., for all $s$ with a fixed radius $\widehat{r}$ and for all $r \leq \widehat{r}$ with a fixed $\widehat{s})$. We show that this information is sufficient to guarantee reconstructability modulo reparametrizations, translations, and rotations.

Lemma 28. Assume that $\gamma$ is TGL for $\hat{r}$ (and thus all $r \leq$ $\widehat{r})$. Then if one knows $g(s, r), g_{s}(s, r)=\partial g(s, r) / \partial s$, and $g_{r}(s, r)=\partial g(s, r) / \partial r$ for $(s, r) \in([0, L] \times\{\widehat{r}\}) \cup(\{\widehat{s}\} \times(0, \widehat{r}])$, one can reconstruct $\gamma(s) \in \mathbb{R}^{2}$ for all $s \in[0, L]$ modulo reparametrizations, translation, and rotations (see Figure 10).

Proof. As was shown in Section 4, $g_{r}$ gives us the length of the arc $\partial D(s, \widehat{r}) \cap \Omega$ and $g_{s}$ tells us precisely what position this arc is along $\partial D(s, \widehat{r})$ with respect to the direction $\gamma^{\prime}(s)$. The assumption of TGL for $r=\widehat{r}$ implies TGL for $0<$ $r<\widehat{r}$ (see Remark 15) and this implies that $\gamma$ has the 2arc property and transverse intersections with $\partial D(s, r)$ for all

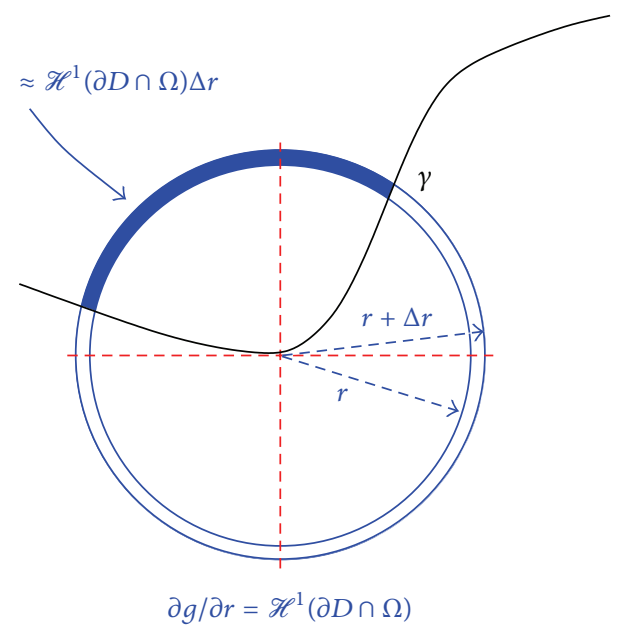

FIGURE 8: Deriving $\partial g / \partial r$ as the arclength of the circular segment.

disks corresponding to $(s, r) \in([0, L] \times\{\widehat{r}\}) \cup(\{\widehat{s}\} \times[0, \widehat{r}])$. Since we care only about reconstructing a curve $\gamma$ isometric to the original curve, we choose $\gamma(\widehat{s})=(0,0) \in \mathbb{R}^{2}$ and $\gamma^{\prime}(\widehat{s})=(1,0)$. Taken together, $g_{s}(\widehat{s}, r)$ and $g_{r}(\widehat{s}, r)$ locate both points in $\partial D(\widehat{s}, r) \cap \gamma$ for all $r \in[0, \widehat{r}]$. This yields $\gamma \cap D(\gamma(\widehat{s}), \widehat{r})$. Now, simply increase $s$, sliding the center of a disk of radius $\widehat{r}$ along $\gamma \cap D(\gamma(\widehat{s}), \widehat{r})$, using $g_{r}(s, \widehat{r})$ to find the element of $\gamma \cap D(\gamma(s), \widehat{r})$ outside $D(\gamma(\widehat{s}), \widehat{r})$, and using the fact that the other element of $\gamma \cap D(\gamma(s), \widehat{r})$ is inside $D(\gamma(\widehat{s}), \widehat{r})$ and known. This process can be continued until the entire curve is traced out in $\mathbb{R}^{2}$.

\section{TCGL Polygon Is Reconstructible from $g_{r}$ and $g_{s}$ without Tail}

Theorem 29. For a tangent-cone graph-like polygon $\Omega$, knowing $g(s, r), g_{r}(s, r)$, and $g_{s}(s, r)$ for all $s \in[0, L)$ and $a$ particular $r$ for which $\partial \Omega$ is tangent-cone graph-like is sufficient to completely determine $\Omega$ up to translation and rotation; that is, one can recover the side lengths and angles of $\Omega$.

Proof. For a given $s$ and $r$ where $g_{r}$ and $g_{s}$ exist, we can use them to obtain $r\left(\theta_{2}-\theta_{1}\right)$ as the length of the circular arc between the entry and exit points by Lemma 26 and $r\left(\sin \theta_{2}-\sin \theta_{1}\right)$ as the difference in heights of the entry and exit points by Lemma 27.

We wish to recover $\theta_{1}$ and $\theta_{2}$ from these quantities. Note that if $\left(\theta_{1}, \theta_{2}\right)=\left(\phi_{1}, \phi_{2}\right)$ is one possible solution, then so is $\left(\theta_{1}, \theta_{2}\right)=\left(2 \pi-\phi_{2}, 2 \pi-\phi_{1}\right)$ and so solutions always come in pairs.

We can imagine placing a circular arc with angle $g_{r} / r$ on our circle and sliding it around until the endpoints have the appropriate height difference, yielding $\theta_{1}$ and $\theta_{2}$. Note that since $\Omega$ is tangent-cone graph-like, one endpoint must be on the left side of the circle and the other must be on the right and we cannot slide either endpoint to or beyond the vertical line through the center of the circle.

Therefore, as we slide the right endpoint down, the left endpoint slides up so that the height difference as a function 


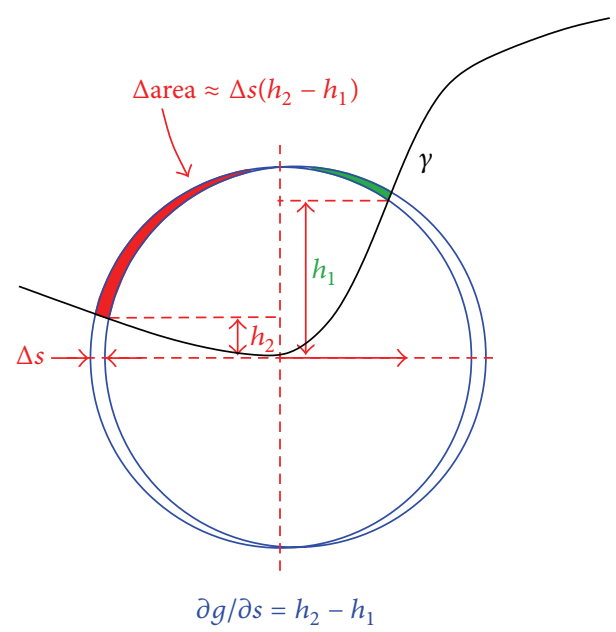

Figure 9: Deriving $\partial g / \partial s$ as the difference in heights of the entry and exit points.

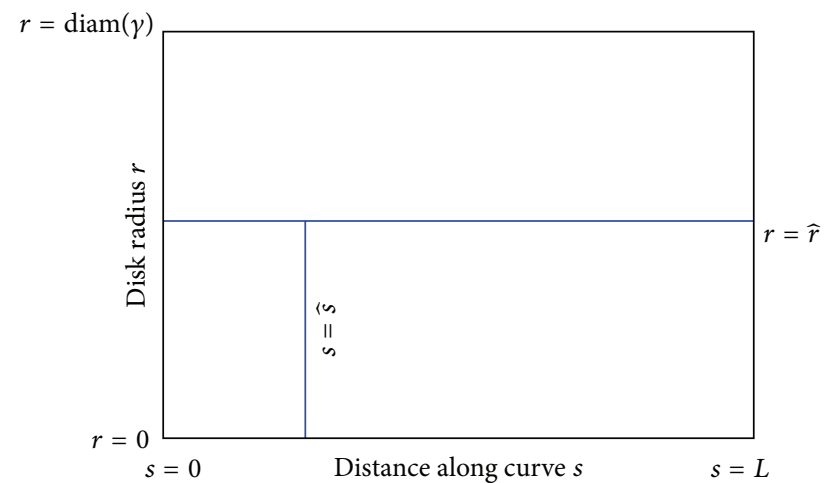

FIGURE 10: T-like data: we restrict the domain of $g(s, r)$ to a fixed radius $\widehat{r}$ plus any vertical segment from $r=0$ to $r=\widehat{r}$.

of the slide is strictly monotonic. Therefore, the slide that gives us $\theta_{1}$ and $\theta_{2}$ is unique for a given starting arc placement. However, there are two starting arc placements: the first calls the angle for the right endpoint $\theta_{1}$ and the left endpoint $\theta_{2}$ (so the interior of $\Omega$ is "up" in the circle) and the second swaps these (so the interior of $\Omega$ is "down"). Since we have adopted the convention that $\partial \Omega$ is traversed in a counterclockwise direction (so the interior of $\Omega$ is up in the circles), we therefore pick the first option; this gives us a unique solution for $\theta_{1}$ and $\theta_{2}$.

This procedure works whenever $g_{r}$ and $g_{s}$ exist which is certainly true whenever the density disk does not touch a vertex of $\Omega$ either at its center or on its boundary because if we avoid these cases, then there is only one graph-like orientation to deal with and $\partial \Omega$ is $C^{\infty}$ for all the points that enter into the computation. In fact, with a moment's thought, we can make a stronger statement than this: $g_{r}$ always exists and $g_{s}$ exists as long as the center of the density disk is not a vertex of the polygon.

We can identify the $s$ values at which $g_{s}(s, r)$ does not exist to obtain the arc length positions of the vertices (and therefore obtain side lengths). For a given $s$ corresponding to a vertex, we can find $g_{r}$ and the one-sided derivatives $g_{s-}$ and $g_{s+}$. These correspond to the graph-like orientations required by the polygon sides adjacent to the current vertex.

Referring to Figure 11, the one-sided derivatives along with the argument at the beginning of the proof yield the angles $\theta_{1}, \theta_{2}, \phi_{1}$, and $\phi_{2}$. Thus, we can calculate $\psi=\theta_{1}-\phi_{1}$ which means that the polygon vertex at $s$ has angle $\pi-\psi$.

Doing this for all $s$ corresponding to vertices, we can determine all of the angles of the polygon. With the side lengths identified earlier, this completely determines the polygon $\Omega$ up to translation and rotation.

\section{Simple Closed Curves Are Generically Reconstructible Using Fixed Radius Data}

We will assume that $\gamma$ is TGL for the radius $\widehat{r}$. We will also assume that we know the first, second, and third derivatives of $g(s, r)$ for $r=\widehat{r}$. Under these assumptions, $\gamma$ is generically reconstructible. By generic we mean the admittedly weak condition of density-reconstructible curves are $C^{1}$ dense in the space of $C^{2}$ simple closed curves.

Theorem 30. Define $\mathbb{G} \equiv\left\{\gamma \mid \gamma\right.$ as a $C^{2}$ simple closed curve and TGL for $r=\widehat{r}$ \}. Suppose that, for $r=\hat{r}$, for all $s \in[0, L]$, and for each $\gamma \in \mathbb{G}$, one knows the first-, second, and third-order partial derivatives of $g_{\gamma}(s, r)$. Then the set of reconstructible $\gamma \in \mathbb{G}$ is $C^{1}$ dense in $\mathbb{G}$ where reconstructability is modulo reparametrization, translation, and rotation.

Proof. In Section 4 we showed that $\partial g(s, r) / \partial r=r\left(\theta_{2}-\theta_{1}\right)$ and $\partial g(s, r) / \partial s=r\left(\sin \left(\theta_{2}\right)-\sin \left(\theta_{1}\right)\right)$, where the notation is as in Figure 6. Because $\gamma$ is TGL, we can solve for $\theta_{1}$ and $\theta_{2}$ from these two derivatives as in the proof of Theorem 29.

Claim 1. The following equations hold: $\partial^{2} g(s, r) / \partial r^{2}=\theta_{2}-$ $\theta_{1}+r\left(\partial \theta_{2} / \partial r-\partial \theta_{1} / \partial r\right)$ and $\partial^{2} g(s, r) / \partial r \partial s=\sin \left(\theta_{2}\right)-\sin \left(\theta_{1}\right)+$ $r\left(\cos \left(\theta_{2}\right)\left(\partial \theta_{2} / \partial r\right)-\cos \left(\theta_{1}\right)\left(\partial \theta_{1} / \partial r\right)\right)$.

Proof of Claim 1. Simply differentiate the expressions we already have for $\partial g(s, r) / \partial r$ and $\partial g(s, r) / \partial s$.

We wish to express this in terms of $v_{1}$ and $v_{2}$. Note that if we expand the circle radius by $\Delta r$, the right exit point $s_{+}(s)$ moves approximately (i.e., considering first-order terms only) a distance of $k \equiv \Delta r \sec \left(\nu_{1}\right)$ (so $\partial k / \partial r=\sec \nu_{1}$, a fact we will use later to compute curvature). Therefore,

$$
\begin{aligned}
\frac{\partial \theta_{1}}{\partial r}=\lim _{\Delta r \rightarrow 0}( & \left(\arctan \left(\frac{r \sin \theta_{1}+k \sin \left(\theta_{1}+v_{1}\right)}{r \cos \theta_{1}+k \cos \left(\theta_{1}+v_{1}\right)}\right)-\theta_{1}\right) \\
& \left.\times(\Delta r)^{-1}\right) .
\end{aligned}
$$

Straightforward techniques yield $\partial \theta_{1} / \partial r=\tan \nu_{1} / r$ and a similar calculation shows that $\partial \theta_{2} / \partial r=\tan \nu_{2} / r$. 

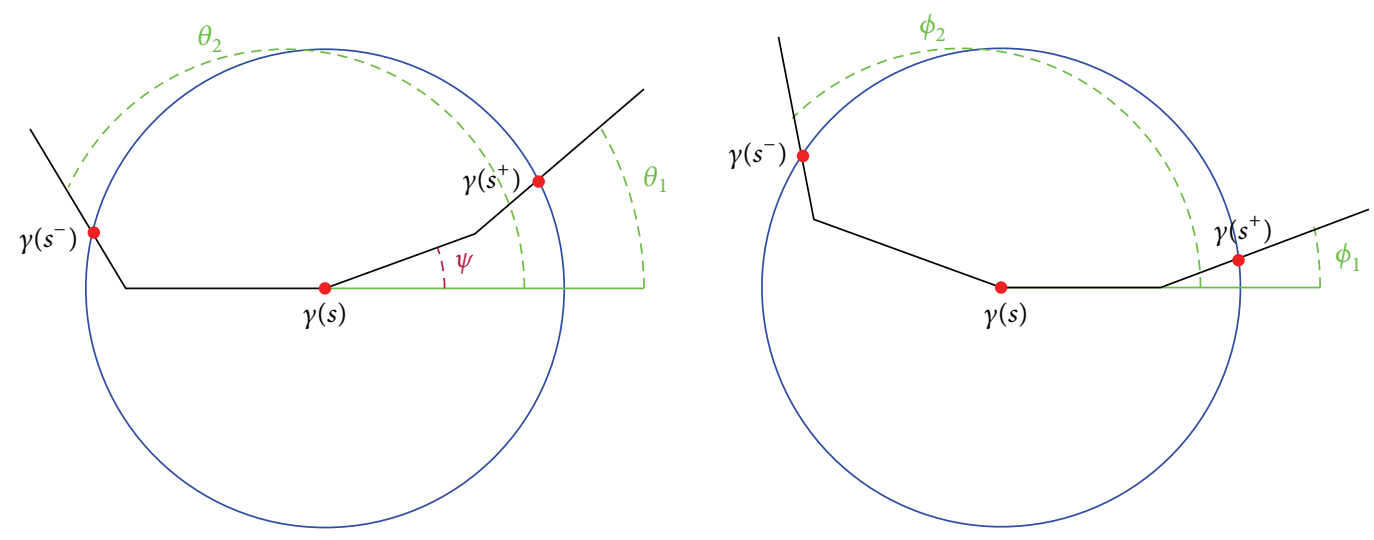

FIGURE 11: Using $g_{s_{-}}$and $g_{s+}$ to obtain the polygon angle at $s$.

Therefore, rewriting the second derivatives of $g(s, r)$ in terms of $\nu_{1}$ and $\nu_{2}$, we get

$$
\begin{gathered}
\frac{\partial^{2} g(s, r)}{\partial r^{2}}=\theta_{2}-\theta_{1}+\tan \left(\nu_{2}\right)-\tan \left(\nu_{1}\right), \\
\frac{\partial^{2} g(s, r)}{\partial r \partial s}=\sin \left(\theta_{2}\right)-\sin \left(\theta_{1}\right)+\cos \left(\theta_{2}\right) \tan \left(\nu_{2}\right) \\
-\cos \left(\theta_{1}\right) \tan \left(\nu_{1}\right) .
\end{gathered}
$$

Using these 2 derivatives, together with the previous two, we can solve for $\nu_{1}=\arctan \left(r\left(\partial \theta_{1} / \partial r\right)\right)$ and $v_{2}=\arctan \left(r\left(\partial \theta_{1} / \partial r\right)\right)$ whenever $\cos \left(\theta_{1}\right) \neq \cos \left(\theta_{2}\right)$. Since we are assuming that the curve is a simple closed curve, $\cos \left(\theta_{1}\right) \neq \cos \left(\theta_{2}\right)$ is always true.

Claim 2. Knowing $\partial^{3} g(s, r) / \partial r^{3}$ and $\partial^{3} g(s, r) / \partial r^{2} \partial s$ gives us $\kappa\left(s^{+}(s)\right)$ and $\kappa\left(s^{-}(s)\right)$, the curvatures of $\gamma$ at $s^{+}(s)$ and $s^{-}(s)$.

Proof of Claim 2. Computing, we get

$$
\begin{aligned}
\frac{\partial^{3} g(s, r)}{\partial r^{3}}= & \frac{\partial \theta_{2}}{\partial r}-\frac{\partial \theta_{1}}{\partial r}+\sec ^{2}\left(\nu_{2}\right) \frac{\partial \nu_{2}}{\partial r}-\sec ^{2}\left(v_{1}\right) \frac{\partial \nu_{1}}{\partial r} \\
\frac{\partial^{3} g(s, r)}{\partial r^{2} \partial s}= & \cos \left(\theta_{2}\right) \frac{\partial \theta_{2}}{\partial r}-\cos \left(\theta_{1}\right) \frac{\partial \theta_{1}}{\partial r} \\
& -\sin \left(\theta_{2}\right) \frac{\partial \theta_{2}}{\partial r} \tan \left(v_{2}\right)+\sin \left(\theta_{1}\right) \frac{\partial \theta_{1}}{\partial r} \tan \left(v_{1}\right) \\
& +\cos \left(\theta_{2}\right) \sec ^{2}\left(v_{2}\right) \frac{\partial v_{2}}{\partial r} \\
& -\cos \left(\theta_{1}\right) \sec ^{2}\left(v_{1}\right) \frac{\partial \nu_{1}}{\partial r}
\end{aligned}
$$

Since $v_{2}^{\prime} \equiv \partial v_{2} / \partial r$ and $v_{1}^{\prime} \equiv \partial v_{1} / \partial r$ are the only unknowns, we end up having to invert

$$
\left[\begin{array}{cc}
1 & -1 \\
\cos \left(\theta_{2}\right) & \cos \left(\theta_{1}\right)
\end{array}\right]
$$

again and this is always nonsingular, giving us $\nu_{1}^{\prime}$ and $v_{2}^{\prime}$ as a function of $s$, the coordinate of the center of the disk.
Relative to the horizontal, the angle of the curve at $s^{+}(s)$ is $\theta_{1}+v_{1}$ so the rate of change in angle as we expand the circle is $\left(\partial \theta_{1} / \partial r\right)+v_{1}^{\prime}$. Recalling that rate of movement of this exit point as we expand the circle is given by $\partial k / \partial r=$ $\sec \nu_{1}$, we have that the curvature is given by $\kappa\left(s^{+}(s)\right)=$ $(\partial k / \partial r)\left(\partial \theta_{1} / \partial r+v_{1}^{\prime}\right)=\sec \nu_{1}\left(\partial \theta_{1} / \partial r+v_{1}^{\prime}\right)$. Similarly, $\kappa\left(s^{-}(s)\right)=\sec \left(\nu_{2}\right)\left(\partial \theta_{2} / \partial r+v_{2}^{\prime}\right)$.

Claim 3. Generically, we can deduce $s^{+}(s)$ from knowledge of $v_{1}(s), v_{2}(s), \theta_{1}(s)$, and $\theta_{2}(s)$.

Proof. We outline the proof without some of the explicit constructions that follow without much trouble from the outline. We have that $\theta_{1}\left(s^{-}(s)\right)+\nu_{1}\left(s^{-}(s)\right)=\pi-\theta_{2}(s)-\nu_{2}(s)$ and $\theta_{1}(s)+\nu_{1}(s)=\pi-\theta_{2}\left(s^{+}(s)\right)-\nu_{2}\left(s^{+}(s)\right)$. All four of these quantities (the left- and right-hand sides of each of the 2 equations) are the turning angles between the tangent to the curve at the center of the disk and the tangent to the curve at a point $r$ away from the center of the disk.

Now we use this correspondence between the $\theta+v$ curves to solve for $s^{-}(s)$ and $s^{+}(s)$. But these curves can differ by a homeomorphism of the domain. Thus, we can only find the correspondence if there is a distinguished point on those curves as well as no places where the values attained are constant. The turning angle curves, having isolated critical points and a unique maximum or minimum, are enough.

To get isolated extrema, start by approximating the curve $\gamma$ with another one $\hat{\gamma}$ that agrees in $C^{1}$ at a large but finite number of points $\left\{s_{i}\right\}_{i=1}^{N}$ (i.e., agrees in tangent direction as well as position) and has isolated critical points in the derivative of the tangent direction. Now perturb $\hat{\gamma}$ to one that is $C^{1}$ close (but not $C^{2}$ close) by using oscillations about the curve so that the 2 nd and 3rd derivatives are never simultaneously below the bounds on the 2nd and 3rd derivatives of the curve we started with. We do this in a way that alternates around the curve. See Figure 12. In a bit more detail, suppose that $\max \left\{d^{2} \widehat{\gamma} / d s^{2}, d^{3} \hat{\gamma} / d s^{3}\right\}<L_{1}$. Choose a starting point on the curve; $s=0$ works. Now begin perturbing $\widehat{\gamma}$ at the point $s_{\widehat{r}}$ in the positive $s$ direction such that $\left|\widehat{\gamma}\left(s_{\widehat{r}}\right)-\widehat{\gamma}(0)\right|=\widehat{r}$. We name the newly perturbed curve $\widehat{\hat{\gamma}}$ and we keep $L_{1}<\max \left\{d^{2} \widehat{\hat{\gamma}} / d s^{2}, d^{3} \widehat{\hat{\gamma}} / d s^{3}\right\}<L_{2}$. 


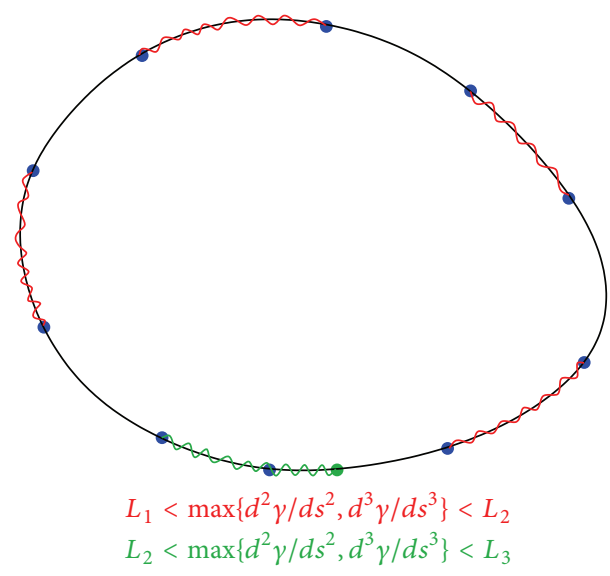

FIGURE 12: In this schematic figure, we illustrate the alternating perturbation around the curve, keeping the curve $C^{1}$ close to and messing with the second and third derivatives to eliminate any critical points other than isolated maxima and minima. Here, the perturbation is of course greatly exaggerated.

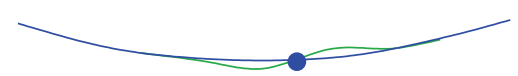

FIGURE 13: A twist perturbation. Notice that if the twist is applied precisely at a global max of the turning angle (as measured by the tangent here and the one lagging it in $s$ ), we will increase the turning angle there and will end up with a unique global maximum.

We continue perturbing until we have reached $s_{2 r}$ defined by $\left|\widehat{\gamma}\left(s_{2 \widehat{r}}\right)-\widehat{\gamma}\left(s_{\widehat{r}}\right)\right|=\widehat{r}$. We begin perturbing again when we reach $s_{3 \widehat{r}}$. Continue in this fashion around $\widehat{\gamma}$. The last piece, shown in green in the figure, will require a perturbation that is distinct in size due to the fact that it will interact with the perturbation that starts at $s_{\widehat{r}}$. On this last piece, we enforce $L_{2}<\max \left\{d^{2} \widehat{\hat{\gamma}} / d s^{2}, d^{3} \widehat{\hat{\gamma}} / d s^{3}\right\}<L_{3}$. All these perturbations can be chosen with isolated singularities in derivatives, thus giving us $\theta+\nu$ curves that are monotonic between isolated singularities. (In fact, we might as well choose all perturbations to be piecewise polynomial perturbations. This immediately gives us the isolated singularities and monotonicity that we want.)

Finally, if there is not a distinct maximum, we can choose one of the maxima and add a small twist to the curve at that point. See Figure 13. The idea is that a small twist, applied to leading edge of the tangents we are comparing to get the turning angle, will increase the angle most at the center of the twist. If this corresponds to a nonunique global maximum, we end up with a unique global maximum.

Now the correspondence scheme works. That is, we know that the global maximums must match, and because the turning angle curves are monotonic between isolated critical points, we can find the homeomorphisms in $s$ that move the turning angle curves into correspondence.
Taken together, the last two claims give us the curvature as a function of arclength. This determines $\gamma$ up to translations and rotations.

\section{Numerical Experiments}

In this section, we consider a numerical curve reconstruction for the situation in which $g(s, r)$ is known for a given radius $r$ but no derivative information is available. This reconstruction is more strict than the scenarios of Sections 57. Our motivation is to explore whether any $\gamma$ can be uniquely and practically reconstructed with this limited information.

We consider $\gamma_{a}(\bar{s}) \in \mathscr{P}^{N}$, the set of simple polygons of $N$ ordered vertices $\left\{\left(x_{1}, y_{1}\right), \ldots,\left(x_{N}, y_{N}\right)\right\}$ parameterized by the set $\left\{\bar{s}_{k}\right\}_{k=1}^{N}$ with $\bar{s}_{k}=k / N$, as

$$
\begin{aligned}
& x_{k}=\sum_{j=0}^{m-1} a_{1, j} \cos \left(\frac{2 \pi j \bar{s}_{k}}{N}\right)+a_{2, j} \sin \left(\frac{2 \pi j \bar{s}_{k}}{N}\right), \\
& y_{k}=\sum_{j=0}^{m-1} a_{3, j} \cos \left(\frac{2 \pi j \bar{s}_{k}}{N}\right)+a_{4, j} \sin \left(\frac{2 \pi j \bar{s}_{k}}{N}\right),
\end{aligned}
$$

for some coefficients $a_{i, j} \in \mathbb{R}$. In this way, the polygon $\gamma$ is a discrete approximation of a $C^{\infty}$ curve. The sides of $\gamma_{a}(\bar{s})$ are not necessarily of equal length.

We take the vector signature $g_{a}(\bar{s}, r) \in \mathbb{R}^{N}$ to be the discrete area densities of $\gamma_{a}(\bar{s})$ computed at each vertex. Given such a signature for fixed radius $r$ and fixed partition $\bar{s}$, we seek $a^{*}$ satisfying

$$
\begin{gathered}
a^{*} \in \underset{b \in \mathbb{R}^{4 m}}{\arg \min }\left\|g_{b}(\bar{s}, r)-g_{a}(\bar{s}, r)\right\|_{2}^{2} \\
\text { s.t. } \gamma_{b} \in \mathscr{P}^{N} .
\end{gathered}
$$

Equation (21) represents a nonlinearly constrained optimization problem with continuous nonsmooth objective. The constraint ensures that polygons are simple though any optimal reconstruction $\gamma_{a^{*}}$ is not expected to lie on the feasible region boundary except in cases of noisy signatures. This approach to reconstructing curves seeks a polygon that matches a given discrete signature, rather than an anytic sequential point construction procedure.

We use the direct search ORTHOMADs algorithm [7] to solve this problem. MADS class algorithms do not require objective derivative information $[7,9]$ and converge to second-order stationary points under reasonable conditions on nonsmooth functions [10]. We implement our constraint using the extreme barrier method [11] in which the objective value is set to infinity whenever constraints are not satisfied. We utilize the standard implementation with partial polling and minimal spanning sets of $4 m+1$ directions.

We performed a series of numerical tests using the synthetic shamrock curve shown in black in the upper portion of Figure 14. This curve is given as a polygon in $\mathscr{P}^{256}$ with discretization coefficients $a \in \mathbb{R}^{4 \times 20}(m=20)$. A sequence of reconstructions was performed with all integer values $8 \leq m \leq 20$. The $m=8$ reconstruction 
begins with initial coefficients, $a_{i, j}$, which determine a regular 256-gon with approximately the same interior area as the shamrock (as determined by the signature $g_{a}(\bar{s}, r)$. In particular, the value(s) $a_{i, j}$ supplied initially are those which define the best fit circle $(m=1)$, which can be computed directly. That is, only $a_{1,0}$ and $a_{4,0}$ are nonzero. Subsequent reconstructions begin with initial coefficients optimal to the previous relatively coarse reconstruction. Curve reconstructions for $m=12$ (blue) and $m=18$ (red) are compared to the shamrock in the upper portion of Figure 14. Reconstructions for $m \geq 20$ are visually indistinguishable from the actual curve and are not shown. Corresponding area density signatures are shown in the lower portion of Figure 14. A representative disk of radius $r$ is shown in green along with corresponding location in the signature; note that the shamrock is not tangent-cone graph-like with this radius.

When comparing and interpreting the shamrock curves, it is important to note that the scale of the curves is determined entirely by the fit parameters $a_{i, j}$. On the other hand, as the density signature is independent of curve rotation, the rotation is eyeball adjusted for easy visual comparison. Also note that the two-arc property does not hold for this example so our reconstructability results do not apply. The accuracies of both the curve reconstruction and area density signature fit suggest that somewhat more general reconstructability results hold. In particular, we speculate that general simple polygons may be reconstructible from $g(s, r)$ for fixed $r$ and no derivative information.

\section{Conclusions}

We have studied the integral area invariant with particular emphasis on the tangent-cone graph-like condition. In particular, we have shown that all TCGL polygons and a $C^{1}$ dense set of $C^{2}$ TGL curves are reconstructible using only the integral area invariant for a fixed radius along the boundary and its derivatives.

We also showed that TCGL boundaries can be approximated by TCGL polygons, determined what the derivatives represented, and commented on other sets of data sufficient for reconstruction (namely, both $\mathrm{T}$-like and all radii in a neighborhood of 0 ).

These reconstructions are all modulo translations, rotations, and reparametrizations. The arc length parameterization plays a special role here since any two such parameterizations of a boundary will differ only by a shift and can easily be placed into correspondence. The situation becomes more complicated in higher dimensions as boundaries are no longer canonically parameterized by a single variable which is a fundamental assumption of our results and methods. It is not immediately obvious how to resolve the issues created by higher dimensions except that it may be possible to modify some of the machinery to work with star convex regions which restore some semblance of canonical representation.

Another space which is open for further development is that of reconstruction algorithms. This is doubly true since
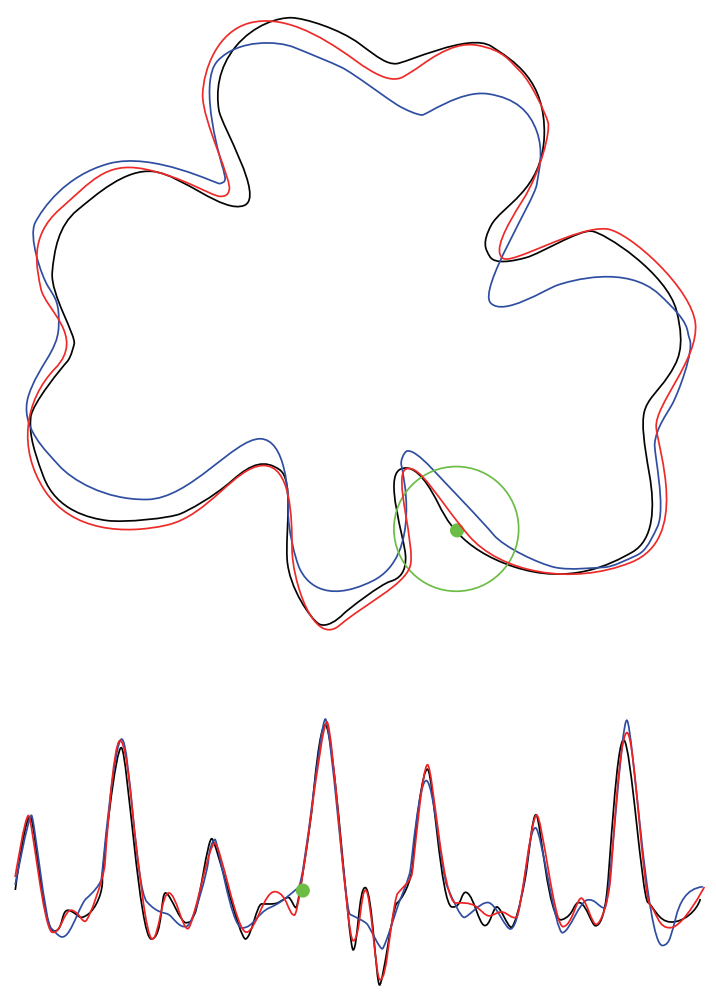

FIGURE 14: Shamrock reconstruction: comparing the original curve with those found for $m=12$ and $m=18$. Curves for $m \geq 20$ are visually indistinguishable from the original curve. The shape signatures are given at the bottom.

our theoretical reconstructions are unstable and the numerical examples in the present work do not have guaranteed reconstruction. However, even without these guarantees, the numerical examples hint at more expansive reconstructability results.

\section{Appendix}

\section{A. Easy Reconstructability}

For completeness, we include a short proof of the fact that knowing $g(s, r)$ for all $s$ and $r$ very easily gives us reconstructability. This follows from the fact that knowing the asymptotic behavior of $g(s, r)$ as $r \rightarrow 0$ for any $s$ gives us $\kappa(s)$. That in turn implies that knowing $g(s, r)$ in any neighborhood of the set $(s, r) \in[0, L] \times\{r=0\}$ also gives us $\kappa(s)$ and therefore the curve.

Theorem A.1. Suppose that $\partial \Omega$ is $C^{2}$ and there exists $\epsilon>0$ such that one knows $g(s, r)$ for all $(s, r) \in[0, L) \times(0, \epsilon)$. This information is enough to determine the curvature of every point on $\partial \Omega$. In particular, if $\gamma:[0, L) \rightarrow \partial \Omega$ is a counterclockwise arclength parameterization of $\partial \Omega$, then $\kappa(\gamma(s))=-3 \pi \lim _{r \rightarrow 0}(\partial / \partial r)\left(g(s, r) / \pi r^{2}\right)$.

Proof. Fix $s \in[0, L)$. If the curvature of $\gamma$ at $s$ is positive, we consider what happens if we replace $\Omega$ with the disk whose 


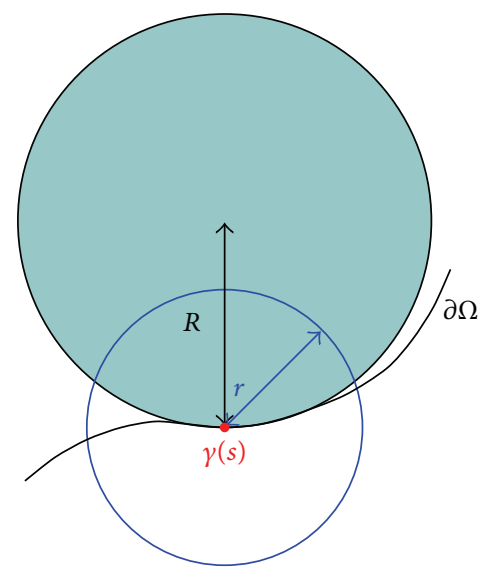

(a)

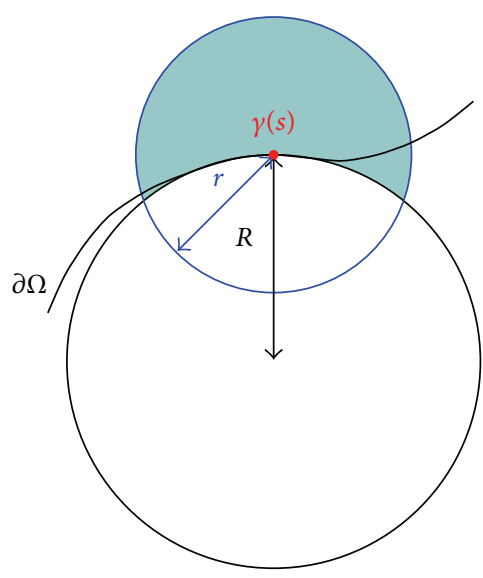

(b)

FIGURE 15: Using the osculating circle as a surrogate for $\partial \Omega$ in the (a) positive and (b) negative curvature cases.

boundary is the osculating circle of $\partial \Omega$ at $\gamma(s)$ (call its radius $R$ ). We have the following expression for the new normalized nonasymptotic density (see Figure 15(a)):

$$
\frac{g(s, r)}{\pi r^{2}}=\frac{1}{\pi r^{2}} \int_{-p}^{p} \sqrt{r^{2}-x^{2}}-\left(R-\sqrt{R^{2}-x^{2}}\right) d x,
$$

where $x=p$ is the positive solution to $\sqrt{r^{2}-x^{2}}=R-$ $\sqrt{R^{2}-x^{2}}$. Differentiating with respect to $r$ and taking the limit as $r$ goes to 0 give us $-1 / 3 \pi R$. That is, for the case where $\Omega$ is locally a disk, the curvature at $\gamma(s)$ is given by $-3 \pi \lim _{r \rightarrow 0}(\partial / \partial r)\left(g(s, r) / \pi r^{2}\right)$.

If the curvature of $\partial \Omega$ at $\gamma(s)$ is negative, we can set up a similar surrogate (see Figure 15(b)) and again obtain that $\kappa(\gamma(s))=-3 \pi \lim _{r \rightarrow 0}(\partial / \partial r)\left(g(s, r) / \pi r^{2}\right)$.

Lastly, this calculation gives the right result in the curvature 0 case when $\partial \Omega$ is locally a straight line ( $\operatorname{so} g(s, r) / \pi r^{2}=$ $\left(1 / \pi r^{2}\right) \int_{-r}^{r} \sqrt{r^{2}-x^{2}} d x=1 / 2$ for sufficiently small $r$ and $\left.-3 \pi \lim _{r \rightarrow 0}(\partial / \partial r)\left(g(s, r) / \pi r^{2}\right)=0\right)$.

For the case where $\partial \Omega$ is not locally a circle or straight line, the corrections to the integrals are of order $O\left(x^{3}\right)$ as $r$ goes to 0 and have no impact on the final answer so the curvature at $\gamma(s)$ is always given by $-3 \pi \lim _{r \rightarrow 0}(\partial / \partial r)\left(g(s, r) / \pi r^{2}\right)$. The available data (the values $g(s, r)$ for all $s \in[0, L)$ and all $r \in(0, \epsilon))$ are sufficient to compute the relevant derivative and limit so we can use this process to determine the curvature of every point on the $C^{2}$ curve $\partial \Omega$.

\section{Conflict of Interests}

The authors declare that there is no conflict of interests regarding the publication of this paper.

\section{Acknowledgments}

The authors would like to thank David Caraballo for introducing them to this topic as well as Simon Morgan and William Meyerson for initial discussions and work on related topics that are not in this paper. This research was supported in part by the National Science Foundation Grant DMS0914809.

\section{References}

[1] S. Manay, B. W. Hong, A. J. Yezzi, and S. Soatto, "Integral invariant signatures", in Proceedings of the 8th European Conference on Computer Vision (ECCV '04), Lecture Notes in Computer Science, pp. 87-99, May 2004.

[2] T. Fidler, M. Grasmair, and O. Scherzer, "Identifiability and reconstruction of shapes from integral invariants," Inverse Problems and Imaging, vol. 2, no. 3, pp. 341-354, 2008.

[3] T. Fidler, M. Grasmair, and O. Scherzer, "Shape reconstruction with a priori knowledge based on integral invariants," SIAM Journal on Imaging Sciences, vol. 5, no. 2, pp. 726-745, 2012.

[4] M. Bauer, T. Fidler, and M. Grasmair, "Local uniqueness of the circular integral invariant," http://arxiv.org/abs/1107.4257.

[5] J. Calder and S. Esedoglu, "On the circular area signature for graphs," SIAM Journal on Imaging Sciences, vol. 5, no. 4, pp. 1335-1379, 2012.

[6] S. Ibrahim, K. Sonnanburg, T. J. Asaki, and K. R. Vixie, "Shapes from non-asymptotic densities," in Proceedings of the SIAM Conference on Imaging Science (IS '10), 2010.

[7] M. A. Abramson, C. Audet, J. E. Dennis Jr., and S. Le Digabel, "OrthoMADS: a deterministic MADS instance with orthogonal directions," SIAM Journal on Optimization, vol. 20, no. 2, pp. 948-966, 2009.

[8] F. Morgan, Geometric Measure Theory: A Beginner's Guide, Academic Press, Burlington, Canada, 4th edition, 2009.

[9] C. Audet and J. E. Dennis Jr., "Mesh adaptive direct search algorithms for constrained optimization," SIAM Journal on Optimization, vol. 17, no. 1, pp. 188-217, 2006.

[10] M. A. Abramson and C. Audet, "Convergence of mesh adaptive direct search to second-order stationary points," SIAM Journal on Optimization, vol. 17, no. 2, pp. 606-619, 2006.

[11] C. Audet, J. E. Dennis Jr., and S. Le Digabel, "Globalization strategies for mesh adaptive direct search," Computational Optimization and Applications, vol. 46, no. 2, pp. 193-215, 2010. 


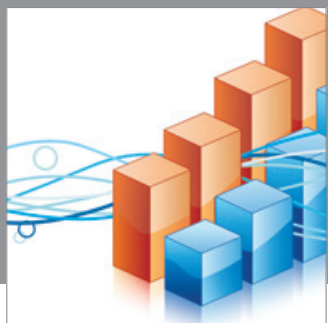

Advances in

Operations Research

mansans

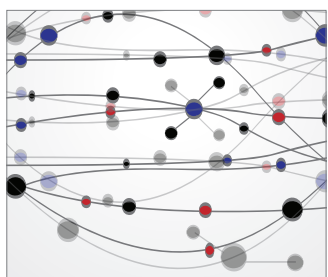

The Scientific World Journal
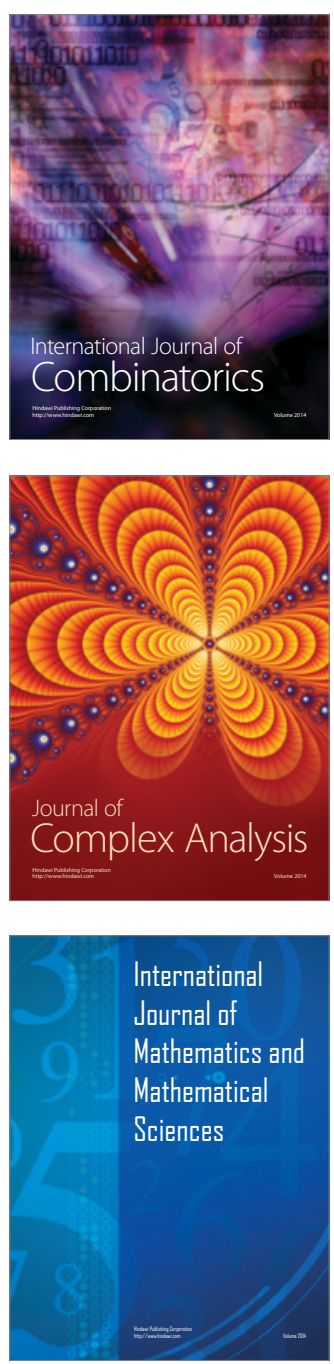
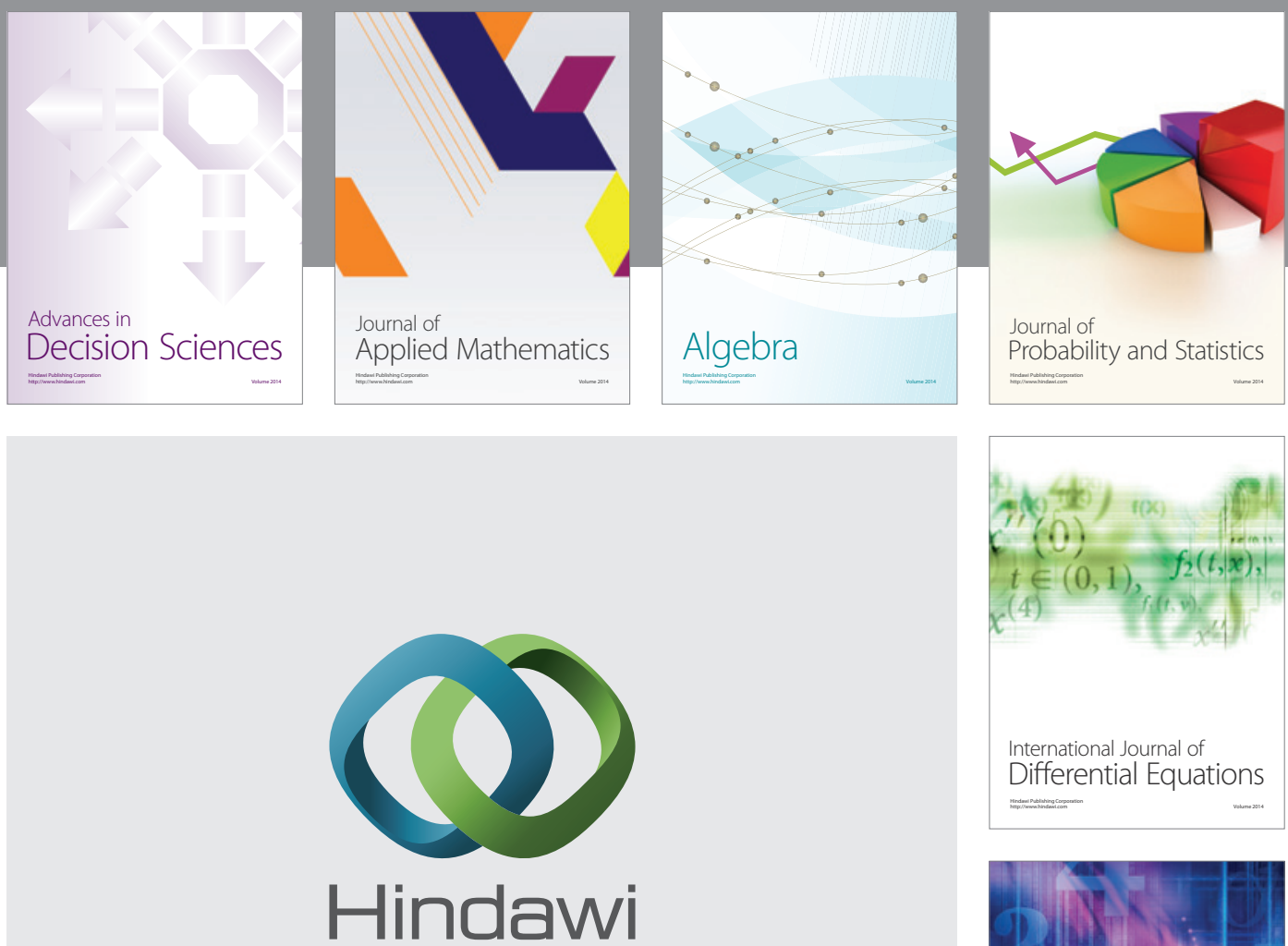

Submit your manuscripts at http://www.hindawi.com
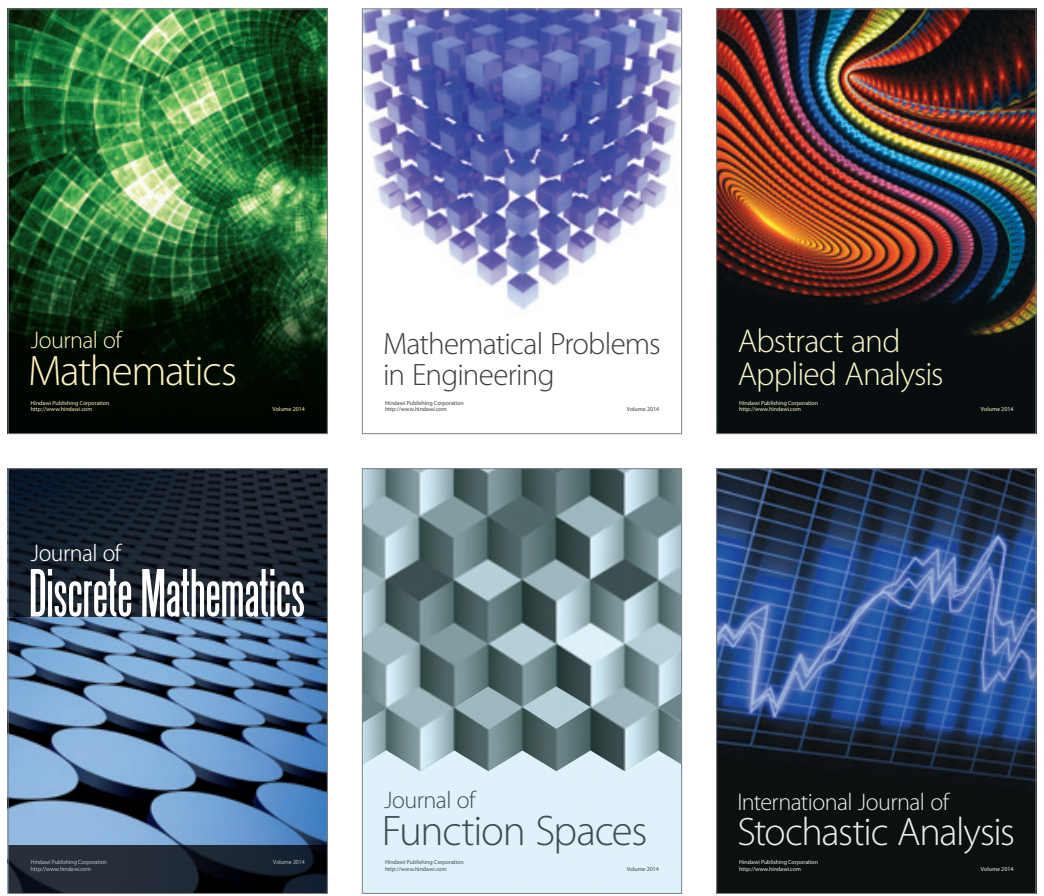

Journal of

Function Spaces

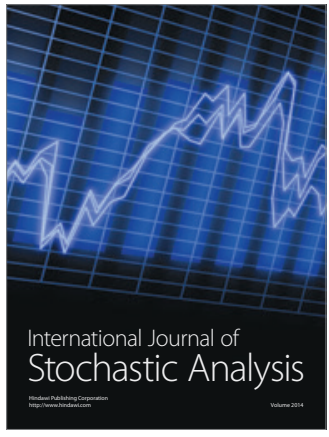

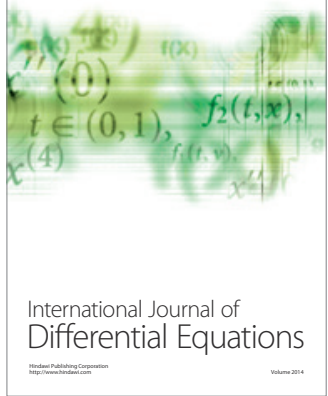
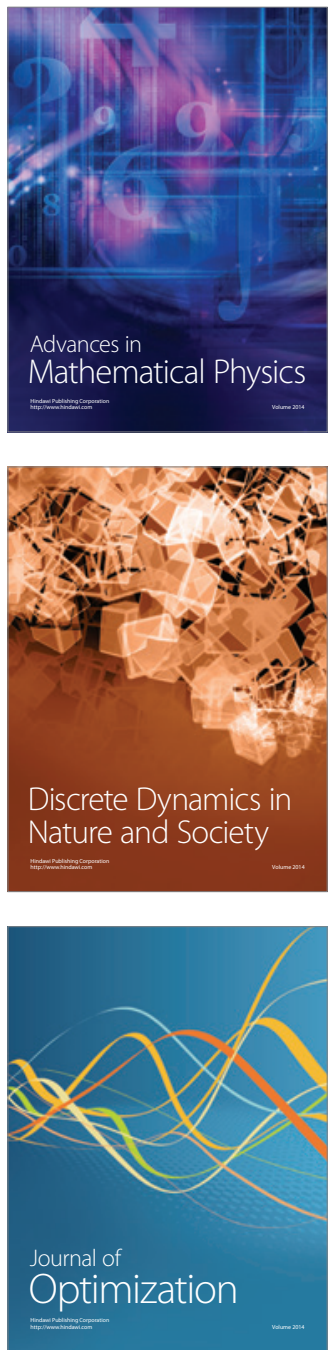